\title{
Nitroxide spin labels and EPR spectroscopy: a powerful association for protein dynamics studies
}

\author{
F. Torricella ${ }^{1}$, A. Pierro ${ }^{2}$, E. Mileo ${ }^{2}$, V.Belle ${ }^{2}$, A.Bonucci ${ }^{12^{*}}$ \\ 1- CERM-Magnetic Resonance Center, Department of Chemistry, University of Florence, via \\ L.Sacconi 6, 50019 Sesto Fiorentino, Italy \\ 2- Aix Marseille Univ, CNRS, BIP, Bioénergétique et Ingénierie des Protéines, IMM, Marseille, \\ France \\ * Correspondance e-mail: bonucci@ cerm.unifi.it \\ abonucci@imm.cnrs.fr
}

\begin{abstract}
Site-Directed Spin Labelling (SDSL) technique consists in the attachment of a paramagnetic label onto a specific position of a protein (or other bio-molecules) and in its subsequent study by Electron Paramagnetic Resonance (EPR) spectroscopy. In particular, continuous wave EPR (cw-EPR) spectra can detect the local conformational dynamics of proteins under various conditions. Moreover pulseEPR experiments on doubly spin-labelled proteins allow measuring distances between spin centres in the 1.5-8 nm range, providing information about the global structure.

This review focuses on SDSL-EPR spectroscopy as a structural biology tool to investigate protein using nitroxide labels. The versatility of this spectroscopic approach for protein structural characterization has been demonstrated through the choice of recent studies. The main aim is to provide a general overview of the technique, in particular for non-experts, in order to spread the applicability of this technique in various fields of structural biology.
\end{abstract}

Keywords: SDSL, EPR, DEER, nitroxide radicals, in-cell EPR

\section{Introduction}

EPR spectroscopy can be successfully employed to detect and characterize different paramagnetic species present in biological systems, such as metal cofactors, Reactive Oxygen Species (ROS) and other molecules which have unpaired electrons in their structures [1-3]. However, the application of this technique can be extended to native diamagnetic samples through the application of Site Directed Spin Labelling (SDSL) methodology [4,5] . 
The SDSL approach is based on the covalent attachment of a paramagnetic tag (called spin label) onto a specific position of a biomolecule (proteins[4-6], DNAs and RNAs [7] as well as lipids[8]), followed by its characterization through continuous wave-EPR (cw-EPR) and/or pulsed dipolar EPR experiments.

Concerning spin labels, a large panel of molecules is currently available: i) ions of transition metals (i.e. Gd(III), Mn(II), etc.)[9,10] (Fig. 1E); ii) nitroxides (Fig. 1 A-D)[11] and iii) trityl-radicals (Fig. $1 \mathrm{~F})[12]$.

Cw-SDSL-EPR experiments are usually carried out at room temperature and thus information about conformational changes, solvent accessibility and interactions between biomolecules can be achieved $[4,6,13]$. Moreover, using pulsed EPR methods, in particular Double Electron-Electron Resonance (DEER), inter-label distances distribution between two spin labels in 1.5-8 nm range (even if distances up to $16 \mathrm{~nm}$ are detectable for fully-deuterated samples) can be measured, providing information concerning large conformational transitions or biomolecules associations[14].

SDSL-EPR is not limited by the size of the molecule under investigation, so it can be applied to various kinds of proteins: from globular [15] to membrane proteins $[16,17]$ whatever its flexibility, and also for intrinsically disordered proteins [18]. In addition, SDSL-EPR spectroscopy is extremely useful to detect minor population states, which are very difficult to reveal with others methods, for example X-ray Crystallography [19,20] and Cryo-electron microscopy [21] .

This review summarizes the principles and the recent progresses of the SDSL-EPR methodological approaches in the investigation of protein structural dynamics, focusing on the use of nitroxide spin labels. Recent examples of both cw-EPR and pulse dipolar spectroscopic studies will be given to underline the strength of this technique for structural characterization of proteins. In addition, the investigation of protein dynamics and conformational changes in cellular environments (in-cell EPR) or in physiological conditions using SDSL-EPR technique with nitroxide spin labels will be also discussed to show one of the current challenging and innovative field of bio-structural EPR spectroscopy.

\section{Nitroxide-based spin labels}

SDSL-EPR methodology, initially introduced by McConnell H.M.[22] in 1965 and successively developed by the work of Hubbell W.L.[5,23,24], is based on the covalent incorporation of a spin label onto a specific site of a protein. Nitroxide labels are heterocyclic (5 or 6-membered ring) free radicals which contain an unpaired electron $(\mathrm{S}=1 / 2)$ in the $\mathrm{N}-\mathrm{O} \bullet$ moiety, stabilized by four bulky groups (generally methyl) on the carbon atoms in $\alpha$-position to nitrogen atom (Fig. 1 A-D)[11,25]. 
By far, nitroxide radicals are the most popular class of spin labels used in SDSL-EPR for structural studies of biomolecules due to their high stability and unique ability to probe local environment[11]. Nitroxide labels can be covalently attached to the side chains of a protein since they are functionalized with specific reactive linkers, such as thiosulfonate, maleimido or iodoacetamide groups (Fig. 1 AD). These groups are introduced during the synthesis onto the heterocycle of nitroxide, in order to react selectively with the thiol group of cysteine residue forming either covalent S-S or C-S bonds (Fig. 2A-B) [4,11]. However, various types of SDSL approaches, involving different labelling sites (such as tyrosine[26], unnatural amino acids [27-29] or peptide synthesis [30,31]), have been developed to expand the applicability of this approach.

The production of cysteine variants is often the starting point of a SDSL-EPR study. This technique usually requires unique residues for site-specific introduction of the spin label, thus any native cysteine needs to be substituted using mutagenesis strategies, except for those that are unreactive to the spin label due to lack of accessibility. For the application of DEER spectroscopy, two cysteine residues (or specific labelling sites) need to be present in the primary sequence in order to measure the dipolar interaction between the paramagnetic centers [14].

In some cases, the use of cysteine as grafting site is not possible since this residue can be involved in disulphide bridges or in a metal cofactor coordination, essential for folding or activity [32]. To circumvent this problem a possibility is to specifically target another residue using for example the nitroxide 4-amino-2,2,5,5-tetramethyl-3-imidazo-line-1-yloxy (NOX) able to be grafted onto tyrosine through a Mannich-type electrophilic aromatic substitution (Fig. 2C) [26]. Another possible strategy relies on the incorporation of an unnatural amino acid (i.e. p-Acetyl-Phenylalanine) into the primary sequence of the protein, using orthogonal $t R N A$ aminoacyl-tRNA synthetase and then labelling with a specific nitroxide tag (i.e. HO-4120) (Fig. 2 D) [27]. Moreover, peptide synthesis and the use of specific nitroxide tags (i.e. 2,2,6,6-tetramethylpiperidine-1-oxyl-4-amino-4carboxylic acid (TOAC) or 4-(3,3,5,5-tetramethyl-2,6-dioxo-4-oxylpiperazin-1-yl)-L-phenylglycine (TOPP)) [30,31] can represent interesting alternatives for the production of nitroxide-labelled samples.

The schematic protocol for the common labelling reaction is depicted in Fig. 3. Initially, the protein is incubated with an excess of a reducing agent (dithiothreitol (DTT) or tris (2-carboxyethyl)phosphine (TCEP), for example) in order to prepare the -SH group for the attachment of the spin label. Once the reagent has been removed, nitroxide label (generally with a 1:10 protein:label molar ratio) is added to the solution and the reaction is kept under gently stirring for at least 1 hour (usually SDSL reactions are performed for 4 hours but longer incubation times up to 24 hours can be required). The reaction can be carried out at room or lower temperatures (i.e. $4^{\circ} \mathrm{C}$ ), depending on protein stability. Spin label amount can be modulated in order to increase the labelling efficiency. Finally, 
the excess of unreacted tag needs to be removed using size-exclusion chromatography (or dialysis technique and centrifugal filters). The cw-EPR spectrum of each fraction of the sample is recorded in order to check the complete elimination of the free label.

\section{General principles of continuous wave-EPR spectroscopy of nitroxide-based spin labels}

EPR spectroscopy is based on the absorption of radiation in the microwave region of the electromagnetic spectrum by paramagnetic species set in an external magnetic field.

To understand the principles behind EPR, a single free electron with $S=1 / 2$ can be used as an example. The two orientations of the electronic spin of the free electron (respectively $M_{s}=+1 / 2$ and $M_{s}=-1 / 2$ ) present the same energy in the absence of an external field. In the presence of a static magnetic field, the magnetic components align parallel or antiparallel to the field leading to spin energy states that are no longer degenerated (Zeeman effect, Fig.4A). The difference in energy between the two levels $(\Delta E)$ can be expressed by:

$$
\Delta E=g \beta B \quad(\text { Eq.1) }
$$

Where $\beta$ is the Bohr magneton $\left(9.24 \times 10^{-24} \mathrm{~J} \mathrm{~T}^{-1}\right)$ and $B$ the external magnetic field. The $g$ factor determines the resonant field position and it describes the electronic configuration and the orientation of the paramagnetic system in the external magnetic field (for a free electron $g \approx 2.0023$ ). Due to a large orbital angular momenta contribution, some samples (i.e organic radical and transition metals) have $g$ factor values different to that reported for a free electron.

In a cw-EPR experiments, the sample is continuously irradiated with microwaves. A cw-EPR spectrum is recorded by linearly varying the external magnetic field and by recording the absorption of the electromagnetic wave. The resonance phenomenon takes place when the emitted energy fits exactly the energy difference between the two energy levels $(\Delta \mathrm{E})$, leading to the equation:

$$
\Delta E=h v=g \beta B \quad(\text { Eq.2) }
$$

Where $h$ is the Planck's constant, $v$ the microwave frequency (i.e $9.8 \mathrm{GHz}$ for X-band, $34 \mathrm{GHz}$ for Q-band and $95 \mathrm{GHz}$ for W-band)

For nitroxide radicals (Fig.4B), the unpaired electron is delocalised over the N-O• group (40\% and $60 \%$ of spin density respectively delocalised on the nitrogen and the oxygen atoms)[33]. The electron spin interacts with the nuclear spin of the ${ }^{14} \mathrm{~N}$ nucleus leading to a so-called hyperfine interaction that 
is responsible for the splitting of each spin state into three sub-states depending on the value of $\mathrm{M}_{\mathrm{I}}$ (1, 0, +1) (Fig. 4A). Since three different transitions are allowed, the EPR spectrum of free nitroxides is composed of three defined lines. A typical X-band $(9.8 \mathrm{GHz})$ isotropic spectrum of a free nitroxide label (MTSL) is reported in Fig. 4C, where the $g$-value is referred as $g_{i s o}$. Since the $p_{z}$ orbital presents an axial symmetry, the electron-nucleus hyperfine interaction (measured by the hyperfine constant A) shows larger values in the direction of molecular $\mathrm{z}$-axis $\left(\mathrm{A}_{\mathrm{x}} \approx \mathrm{A}_{\mathrm{y}} \neq \mathrm{A}_{\mathrm{z}}\right)[11,25]$.

For experiments carried out at X-band $(9.8 \mathrm{GHz})$, this anisotropy of the hyperfine interaction is the key of the SDSL-EPR approach rendering nitroxide spin labels sensitive to their local environment. Due to the partial averaging of this anisotropy, the nitroxide motion is directly detectable from the EPR spectral line shape [5,25]. The overall mobility of the spin label is determined by a combination of the motion of the label itself relative to the peptide backbone, the fluctuations of the $\alpha$-carbon backbone, and the rotational motion of the entire protein. For this reason, the cw-EPR spectral lineshape is sensitive to the local structure and the architecture of the environment surrounding the labelling position $[5,11,25]$. The most informative parameter is the rotational correlational time $\left(\tau_{\mathrm{c}}\right)$, that represents the molecular re-orientation of the label with respect to the magnetic field [34]. A simple way to extract $\tau_{\mathrm{c}}$ from EPR spectra of a labelled protein relies on the measurement of the central line width [35]. However, these approaches could be not sufficient for multi-components spectra coming for example from multi-conformational states of the labelled system under investigation. The best way to extract the dynamic parameters is based on the simulations of the experimental data with a specific software, such as EasySpin [36] or SimLabel (a graphical interface using Easypin) [37].

Conventional X-band EPR spectra are sensitive to nitroxide rotational motions in the range of 0.01 ns to $100 \mathrm{~ns}[11,25,38]$; some examples of EPR spectral shapes of nitroxides with different $\tau_{\mathrm{c}}$ are reported in Fig 5. If the labelling site is solvent exposed or it has high degrees of flexibility, the EPR spectrum is characterized by small line widths (Fig. 5B), since the mobility of the spin label side chain is only partially restricted ( $\left.\tau_{\mathrm{c} .}<1 \mathrm{~ns}\right)$. Contrary, nitroxide tags, interacting with backbone atoms and side chains in the surrounding environment, produce EPR spectra with broader line widths and larger hyperfine constants (Fig.5C). Fully immobilized spin label side chains, buried labelling sites and samples at froze temperatures $(<180 \mathrm{~K})$ are related to spectrum as reported in Fig.5D.

\section{Nitroxide-based spin labels and cw-EPR: a tool for the investigation of protein structure and dynamics in solutions}


Due to the sensitivity for the dynamic events in the $p s-n s$ time range $[4,11,13,38]$, SDSL-EPR spectroscopy can be used to monitor disorder-to-order transitions, induced structural variations and possible conformational modifications arising from the binding processes with partner molecules [3941] . In this section, we will focus on some recent SDSL-EPR studies involving nitroxide labels for the characterization of structural changes, protein-nucleotides and protein-protein interactions mainly through cw-EPR spectroscopy.

An investigation performed on the Sporosarcina pasteuri Urease accessory protein G (Sp-UreG) revealed the implication of the intrinsic disorder of this enzyme on its activity by SDSL-EPR spectroscopy, Circular Dichroism spectroscopy and enzymatic assay [42]. Sp-UreG is a GTPhydrolase enzyme involved in the delivery of nickel and the maturation of urease, a multifunctional toxic protein for bacteria and fungi. Through the labelling with MTSL nitroxide (Fig. 2A) the native cysteine (C68) of Sp-UreG (Fig. 6A), the authors analysed the effect of various additives on the structural conformation of the catalytic region of the protein by recording X-band cw-EPR spectra (Fig. 6C). First, the SDSL-EPR results revealed the coexistence of two different conformations with two distinct values of $\tau_{\mathrm{c}}(0.43 \mathrm{~ns}$ and $5.5 \mathrm{~ns}$ respectively for the sharp and the broad components). These values obtained from experimental spectra simulations indicated the equal presence of a very flexible and a more compact conformation for Sp-UreG in solution. Successively a shift between the two states was detected by adding various folding/unfolding agents. In particular, the cw-EPR spectra in presence of 2,2,2-trifluoroethanol (TFE), trimethylamine N-oxide (TMAO) and sodium dodecyl sulfate (SDS) resulted broader than that recorded for native protein, revealing a more compact conformation for $S p$-UreG. The increase of the percentages of the broad EPR signals and the higher value of $\tau_{c}$ were evidences of the protein conformational shift through a less mobile architecture.

On the other hand, sharper EPR signals, reflecting a more flexible structure, were detected by increasing the temperature and adding guanidinium chloride $(\mathrm{GuHCl})$. A peculiar result was observed by comparing the EPR results with catalytic tests performed in the presence of the same additives (Fig. 6B). Indeed, $S p$-UreG showed to be more active when the flexible component is dominant. The authors demonstrated that this protein is an atypical enzyme, since it requires a discrete flexibility to be catalytically competent.

SDSL-EPR spectroscopy has been applied to characterize the interaction of proteins with other biomacromolecules, such as other proteins[43], nucleotides [44,45] and lipids[40,46]. For example, Norman D.G. et al. studied the interaction of the N-terminal region of T7 enodonuclease I with DNA[47]. This enzyme binds the DNA four-way junction and it plays a central role in the doublestrands breaks reparation. Due to the dynamic behaviour of the N-terminal region, previous X-ray crystallography studies failed to define completely the interaction of endonuclease I with nucleotides. 
Through the incorporation of MTSL spin labels on 10 individual positions of the N-terminal domain (Fig. 6D), the authors studied each labelled mutants with and without DNA in solution. The spectra in the absence of nucleotides (Fig. 6E) resulted sharp (except for position 16 and 29 located in the first helix), revealing a great flexibility of this region while, addition of DNA caused a broadening of the spectra occurring between positions 7 and 14. Taking also advantages from DEER experiments (see section 5 and Fig.10), the authors were able to identify a disorder-to-order transition of the Nterminal region of endonuclease I upon DNA-binding, shedding the light on its implication in the reparation process.

Another interesting approach concerns the characterization of a protein-protein interaction in which the two interacting systems carry respectively a nitroxide label. In the study described by Martinho M. et al.[48], the peculiarity is that while one protein bears a MA-PX nitroxide showing the classic three line spectrum(Fig. 7A), the other interacting protein carries a new synthetized $\beta$-phosphorylated nitroxide label (PP) giving a 6-lines spectrum arising from the supplementary hyperfine interaction with the ${ }^{31} \mathrm{P}$ atom (Fig. 7B). Using this strategy, the authors were able to detect and characterize the interaction between the intrinsically disordered $\mathrm{C}$-term region of the measles virus nucleoprotein ( $\mathrm{N}_{\text {tail }}$, a.a. 401-525) with the $\mathrm{X}$ domain of viral phosphoprotein (PXD, a.a. 459-507) (Fig. 7B). Forming a 1:1 mixture of $\mathrm{N}_{\text {tail }}$ and $\mathrm{P}_{\mathrm{XD}}$ respectively labelled with PP and MA-PX (Fig. 7C), they demonstrated in one experiment that the position 496 of the viral protein is implied in partner binding whereas this is not the case for the labelled position of $\mathrm{N}_{\text {tail }}$ (position 407). In particular, the spectral simulations for the $\mathrm{N}_{\text {tail }} \mathrm{S} 407 \mathrm{C}$, bearing PP label, alone in in presence of the partner showed analogues values of $\tau_{\mathrm{c}}$ and component percentages, indicating that the proteins retained its disordered character also in the complex. Contrary, the $\tau_{\mathrm{c}}$ values of the EPR signatures of PXD K496C labelled with MA-PX increased (about $+2.5 \mathrm{~ns}$ for component 1 and about $+20-23 \mathrm{~ns}$ for component 2 ) in presence of $\mathrm{N}_{\text {tail }}$, evidencing the protein-protein interaction.

Another application of nitroxide spin label for protein investigation is to monitor the solvent accessibility profile with power saturation cw-EPR[4,11]. This method relies on the measure of the collisions between a spin labels grafted onto a protein and a relaxing agents (i.e. metal complex or $\mathrm{O}_{2}$ molecule) from Heisenberg exchange rate, which is proportional to the accessibility parameter (П). Specifically, the relaxing agent interacts with the nitroxide linked to a site of a protein accelerating the relaxation rate of the radical. From an experimental point of view, the EPR spectrum of nitroxide labelled protein is recorded respectively with freely diffused paramagnetic oxygen and with the paramagnetic reagent nickel ethylenediamine-N,N'- diacetic acid (NiEDDA)[49,50]. Since $\mathrm{O}_{2}$ is diffused in apolar environment while NiEDDA is soluble in the bulk water only, details about protein structure or insertion into lipid membrane can be achieved with this approach. As example, Walters 
D.E. et al. [51] used this method to probe the membrane insertion of the pro-apoptotic Bcl-2 family proteins BAK. This protein raised the interest since it showed a role in the mitochondrial cell death pathways, forming large pores in the outer membranes. For this reason, the research group investigated the conformational changes adopted by BAK upon the insertion into lipid membranes, labelling multiple sites of the protein with MTSL (Fig. 2A) and the recording the X-band EPR spectra in solution and in membrane (Fig.8A)., $\Pi$ was obtained in presence of oxygen and NiEDDA for all BAK variants in solution and in lipid environment. In solution, small $\Pi\left(\mathrm{O}_{2}\right)$ and $\Pi(\mathrm{NiEDDA})$ were revealed for residue 55, 132 and 134 since they are located in the protein interior while the other labelling positions showed higher values due their localization on protein surface. The EPR experiments revealed large changes in the conformation for BAK upon the addition of liposomes. In particular, both accessibility parameters for residue 55 were significantly increased compared to the solution state, underlining that this position was exposed to solvent phase after BAK association to membrane (Fig. 8B-C). On the other hand, the labelling sites 134, 146, 154 and 164 showed higher values of $\Pi\left(\mathrm{O}_{2}\right)$ for the samples localized into lipid bilayer than those dissolved in buffer, indicating these positions were buried into membrane core (Fig. 8B-C). All the data obtained from this EPR investigation allowed the authors to provide new insights about BAK structure and its mechanism of membrane insertion (Fig. 8D).

\section{DEER experiments for distance measurements between nitroxide-based spin labels}

Through the incorporation of two nitroxide labels onto specific positions of a biological system, the distances between spin pairs can be achieved performing pulse-EPR experiments [14,52,53] . In general, the distance range detected is between 1.5 and $8 \mathrm{~nm}$, even if measurements up to $16 \mathrm{~nm}$ were achieved with a fully deuterated sample [54] . Different types of pulse techniques are available, such as Double Quantum EPR (DQ-EPR) [52] , Relaxation Induced Dipolar Modulation Enhancement (RIDME) [55], SIngle Frequency TEchnique for Refocusing dipolar coupling (SIFTER) [56] and Double Electron-Electron Resonance (DEER, also called Pulsed ELectron DOuble Resonance PELDOR) [14].

In this review, we will focus on DEER since it is the most commonly used pulsed technique with nitroxide labels. Briefly, the 4-pulses DEER consists in the application of a $\pi / 2-\pi-\pi$ refocused echo sequence with fixed interpulse delays at frequency $v_{A}$ (observer frequency) that excite a part of the spectrum corresponding to the spin population that are observed (often described as spin A). The fourth pulse is a $\pi$ pulse at frequency $v_{B}$ (pump frequency) that inverts the magnetization of another 
part of the spectrum referred as spin B (Fig. 9A). For nitroxide, $v_{B}$ is generally set to a field position corresponding to the maximum of the absorbance spectrum and $v_{A}$ is selected at the low field component of the signal (see Fig. 9B).

By varying the position of this pump pulse along the sequence, the refocused echo intensity results modulated by the electron-electron dipolar coupling $\left(\omega_{\mathrm{AB}}\right)$ between spin A and B (Fig. 9C). This modulation is inversely proportional to $r^{-3}$ (where $r$ is the distance between the spin labels) so that the distribution of distances can be calculated from the experimental DEER traces

Short distances between nitroxide tags produce high frequency oscillations in the relative DEER traces, whereas long distances are characterized by low frequency oscillations. DeerAnalysis software developed by Jeschke G. and co-workers (https://epr.ethz.ch/software.html) is commonly adopted as friendly software for standard processing and analysis of experimental traces and the Tikhonov regularization represents the standard method for distance distribution calculation from data analysis [57,58] (see ref. [14,52,53] to get details about DEER theory and data manipulation).

DEER experiments on nitroxide labelled proteins generally require a sample concentration of $\sim 80$ $100 \mu \mathrm{M}$ in about $15-50 \mu \mathrm{L}$ of a total volume[14] and an EPR spectrometer operating at Q-band (34 $\mathrm{GHz}$ ) is preferred because a higher signal-to-noise ratio is obtained when working at this frequency [59]. One of the major limitations of the implication of the nitroxide labels for DEER experiments is represented by their fast relaxation rate at room temperature. For this reason the measurements are usually performed at cryogenic temperature (around $60 \mathrm{~K}$ ) [11,14,25]. Another limiting factor is represented by the phase memory time $\left(T_{m}\right)$; these spin labels possess a $T_{m}<2 \mu \mathrm{s}$, fixing the maximum of detectable interspin distances around 6-8 $\mathrm{nm}$. The use of deuterated solvent often helps to increase $\mathrm{T}_{\mathrm{m}}$ and consequently the measurable distance $[14,60]$. An alternative strategy relies on the use of a spyrocyclohexyl nitroxide radical, which carries cyclohexyl groups on the $\alpha$-carbon of nitrogen atom of the $\mathrm{N}-\mathrm{O} \cdot$ moiety [61]. These shielding groups influence the $\mathrm{T}_{\mathrm{m}}$ of the spin label, giving higher values than those measured for classical spin label, such as MTSL. Due to this unique property, the spyrocyclohexyl nitroxide radical, in combination with the immobilization of the sample into a water/glycerol matrix, permits to perform DEER experiments up to $160 \mathrm{~K}$.

In order to study multi-subunits systems, membrane proteins or large protein complexes, the incorporation of spin label onto more than two site results impossible to circumvent. Using exclusively a nitroxide-based approaches, the interpretation of pairwise distance distributions results extremely challenging since nitroxides have the same spectroscopic properties [62].To overcome this limitation, the orthogonal-spin labelling strategy demonstrated to be a valid approach. Orthogonal labelling mainly consists in the incorporation of a nitroxide-label and a metal-based (Gd (III), Cu (II), Mn (II)) tag respectively on different sites of the system under investigation [63-66]. Since the 
radicals and the metals have different EPR spectra, the nitroxide-nitroxide and the nitroxide-metal distances can be individually obtained adjusting differently the pump and the observer frequencies in the experimental set up. RIDME sequence represents an interesting alternative to DEER experiment for distance measurements between nitroxides and metal centers [67]. This pulse-EPR technique presents some advantages since it requires a single frequency in the sequence for the detection of stimulated echoes [55]. In order to avoid problems arising from the limitation of the excitation bandwidth, the spins B are not excited by a pump pulse as in the DEER experiments (see Fig. 9A), but the flip of these spins is left to the spontaneous longitudinal relaxation. For these reasons, RIDME is well adapted to measure distances between a fast-relaxing metal tag (high g-anisotropy) and a small-relaxing paramagnetic probe (nitroxide with low g-anisotropy).

A recent example of the application of DEER spectroscopy in the field of structural biology is the work performed by Norman D.G. et al. presented partially in the previous section, focused on the role of N-terminal region of T7 enodonuclease I in DNA-binding [47]. Using the same MTSLlabelled samples previously characterized by cw-EPR spectroscopy (see section 4), the authors measured distances between nitroxide labels of the dimeric form of the protein in the absence and presence of DNA. The DEER traces (Fig. 10C) recorded up to position 12 resulted flat compared to those recorded for the position 14-16, where oscillations were clearly visible. The absence of oscillations is an experimental signature usually observed in DEER experiments with flexible proteins (such as intrinsically disordered proteins). Due to the large number of different conformers, the interference between different DEER traces related to different inter-spin interactions causes the dampening of the oscillation and broad profiles of distance distributions.

These DEER results clearly indicated a great flexibility and a disordered conformation adopted by the N-terminal region, in line with cw-EPR results (see Fig. 6E). On the other hand, the addition of DNA in solution induced a decrease of the distances from residue 8 to residue 14, while smaller variations were observed for the other labelling sites (Fig. 10 B-C). The DEER data, in combination with cw-EPR results, indicates that the $\mathrm{N}$-terminal region adopts a more compact conformation in the DNA-complex, inserting into the grooves at the center of DNA junction. (Fig. 10A)

Another recent example is represented by the study performed on the Vibro cholerae Norm-Vc protein, a membrane protein belonging to the multidrug and toxic compound extrusion family that protect the cell from cytotoxic molecules through an efflux mechanism related to ion electrochemical gradients [68]. The research group labelled with MTSL different sites of the C-terminal (CTD), Nterminal (NTD) and trans-membrane domains (TM) on the periplasmic side of NorM-Vc (Fig. 11 top panel). The study was based on the acquisition of the DEER traces for NorM-Vc alone or in presence of a chemotherapeutic drug doxorubicin (DXR) and sodium ions. Comparing the distance distribution 
obtained from DEER experiments with predicted information coming from the crystallography experiments (Fig. $11 \mathrm{~A}-\mathrm{H}$, black and grey lines respectively), the authors revealed notable differences between the conformation adopted by Norm-Vc in solution and in the X-ray model, suggesting that the NTD cavity in the crystal may be collapsed in solution (Fig. 11 C, D and G). Moreover, the DEER distance profiles for the apo-form showed a broad profile, due to dynamic backbone fluctuations that can be modulated by both $\mathrm{Na}^{+}$and DXR. In particular, sodium ions increased the population of longer distances between spin pairs, underlining a conformational shift through a more stable state (Fig. 11 A-H blue lines). On the other hand, DXR showed an opposite effect since distances shorter than those obtained for the apo-form appeared in the related inter-spin distances profiles (Fig. $11 \mathrm{~A}-\mathrm{H}$ orange lines). All the DEER results obtained for Norm-Vc9 indicated that $\mathrm{Na}^{+}$and DXR induce changes in the membrane protein assembly, favouring the formation of different structural intermediates.

Nowadays, an increasing number of scientific papers about the applications and the developments of this technique demonstrate the strength of DEER methodology in the field of structural biology for the investigation of various biomolecules.

\section{EPR experiments with nitroxide-based spin labels inside cells}

The characterization of protein structure and dynamics inside living cells represents a new frontier in the field of structural biology [69]. The macromolecular crowding, the viscosity and the $\mathrm{pH}$ of the cystosol but also possible specific/unspecific interactions are some of the main factors that can influence the conformations and the dynamics of proteins inside cells [70-72].

NMR [73-75] and FRET [76,77] spectroscopies have already demonstrated that the intracellular medium can relevantly affect the protein structures. SDSL-EPR and DEER spectroscopies represent promising tools to perform structural studies into cellular environments (in-cell EPR) [78]. In the last ten years, an increasing number of new spin labels and EPR techniques for the realization of in-cell EPR experiments have been developed. The diversity of the SDSL-based approaches published so far is evidencing the capability of EPR spectroscopy to study proteins (but also nucleic acids [79,80]) conformations and dynamics in real cellular environments.

One of the main limitations of in-cell EPR spectroscopy with nitroxide-based labels is represented by their bio-reduction leading to the diamagnetic specie (hydroxylamine, $\mathrm{N}-\mathrm{OH}$ ) in intracellular environments. Different physiological reducing agents (i.e. glutathione, NADH, metal-proteins, etc.) can be responsible of this phenomenon [81-83]. Even if the rate of the reaction mainly depends on the molecular structure of the nitroxide tag and the spin concentration inside cells, this process generally results fast (several minutes). 
One possibility is to design new nitroxide-based tags able to resist to bio-reduction [83-85]. In general, these tags are characterized by the presence of bulky-groups carbon in the $\alpha$-carbon of the ${ }^{14} \mathrm{~N}$ atom of the nitroxide moiety, which shield the radical from reducing agents.

Among the different shielding substituents investigated for in-cell studies [86,87], the gem-diethylgroups resulted the most efficient. The spin label reported in Fig. 11A, characterized by the introduction of gem-diethyl-groups and by a maleimido as grafting function, was successfully employed in studying protein structural dynamic in cell extracts [88] and inside X.leavis oocytes [89]. In the work of Katikeyan G. et al. [89], this new nitroxide tag showed a 10\% loss of the EPR signal in one hour in reducing environment (Fig. 12C), whereas a total signal loss was observed with the classic MA-PX label in the same conditions. In addition, the authors demonstrated the ability of the new bioresistant nitroxide to be a good reporter of protein structure and dynamics by studying a chaperone protein (NarJ from E. coli) involved in the biogenesis of nitrate reductase. DEER experiments (Fig. 12 E-F) were performed by microinjecting the X. laevis oocytes (Fig.12D) with doubly-labelled NarJ (Fig. 12 B). The results obtained demonstrated the capability of the new nitroxide for the applications of in-cell EPR experiments, due to its resistance to bio-reduction. Moreover, these efforts open new perspectives for the study of protein dynamics in their natural environments with EPR spectroscopy.

Another critical point for the set-up of in-cell EPR experiments relies on the choice of the strategy for protein internalization into cells. Since this technique requires to label the protein prior its delivery inside cells, the development of approaches for the intracellular incorporation is needed. In the past years, electroporation, osmotic-shock and microinjection have been used for protein delivery into prokaryotic and eukaryotic cells [90]. An alternative way was recently reported by the group of Drescher M. [28], who bio-synthetized a labelled protein directly into the cytosol of bacteria cells combining the genetic encoding of unnatural amino acid in response to the amber stop codon (TAG) by a tRNAPyl/pyrrolysyl-tRNA-synthethase pair in E.coli and a modified lysine carrying a nitroxide moiety. Using thioredoxin (TRX) as model system, the authors demonstrated that the protein expression inside cells reached a sufficient level to be detectable by EPR spectroscopy. However, it was not possible to perform in-cell DEER experiments due to the rapid reduction of the nitroxide in the intracellular environment and the proteolysis of TRX exerted by E.coli enzymes.

SDSL-EPR spectroscopy with nitroxide spin labels can also be used for the investigation of membrane proteins in their native lipid environments. A valid proof is the study performed to characterize the dynamic of the outer-membrane cobalmin transporter BtuB directly in intact E.coli cells [91]. The authors overexpressed into E.coli a variant of BtuB carrying two cysteine mutations respectively into two extracellular loops and then they labelled the protein with MTSL. Several 
experiments were performed to demonstrate that the SDSL reaction was exclusively for the system under investigations. Indeed, E.coli membranes do not contain proteins bearing cysteines and the free nitroxide fraction situated the periplasm and cytoplasm was rapidly reduced by cellular agents. Then, the DEER traces of nitroxide labelled BtuB in the E.coli suspension were recorded in absence and in presence of calcium ions and cyanocobalamin, revealing notable conformational changes of the extracellular loops in these different conditions. The entire project represents one of the first structural changes of a protein observed with SDSL-EPR spectroscopy in its native environment.

Another example of the application of SDSL-EPR spectroscopy for the characterization of membrane proteins in physiological conditions is represented by the study performed on colicin A (ColA) by Klare P.J. and co-workers [92]. In particular, the research group investigated on the oligomerization of the pore-forming toxin ColA in artificial and real E.coli lipid bilayers through the combination of cw-EPR and DEER spectroscopies. Even if the DEER traces presented a low signal-to-noise ratio, the results obtained demonstrated that ColA assumes the same organization in both in-vitro and in intact cells at the native concentration of the membrane protein.

It is important to highlight that various types of $\mathrm{Gd}(\mathrm{III})$-based tags, which are not sensitive to bioreduction, have been demonstrated to be excellent probes to obtain distances in protein localized into different cells [93].

Morevover, also trityl radicals have also been proven to be efficient tags for spin-spin distances measurements inside cells and to be highly-redox stable labels in those environments [94]. However, future improvements on the design of trityl-radical are required to expand its applicability for in-vitro and in-vivo EPR studies on proteins and other macromolecules.

The research based on the investigation of structures and functions of biomolecules in intracellular conditions is a step forward in the field of structural biology, which could permit to enlarge the knowledge about various cellular mechanisms and processes.

\section{Concluding remarks}

This review summarizes the basic principles of SDSL-EPR spectroscopy using nitroxides labels and its application in the field of proteins research. The examples provided about cw-EPR and DEER spectroscopies underline the variety of information that can be achieved with nitroxide-labelled proteins concerning their conformations, dynamics and functions. Moreover, these tags have been demonstrated to be efficient probes for the study of proteins directly inside cells.

The nitroxide labels and the SDSL-EPR spectroscopy have demonstrated to be excellent tools able to provide atomistic information which can be also combined with other methods, playing an important role in field of structural biology. 


\section{Acknowledgments}

A.B. would like to thank Dr. J.L. Neira for the invitation to write this review. A.B. is grateful to "Regione Toscana" for the financial support received for the post-doctoral grant (POR-FSE 20142020). CERM/CIRMMP center of Instruct-ERIC and the Italian Ministry for University research (FOE funding) are gratefully acknowledge by F.T. and A.B.

We are grateful to the EPR facilities available at the French national EPR network RENARD (IR 3443), and at the Aix-Marseille EPR center. We also acknowledge financial support from the Agence

National de la Recherche (contract ANR-18-CE11-0007-01 and ANR-18-CE29-0003 NANODISPRO) and the “Conseil Régional Région Sud” (A.P. PhD Fellowship EJD-2018-2021).

\section{References}

[1] S. Suzen, H. Gurer-Orhan, L. Saso, Detection of reactive oxygen and nitrogen species by electron paramagnetic resonance (EPR) technique, Molecules, 22 (2017) 1-9.

[2] M.M. Roessler, E. Salvadori, Principles and applications of EPR spectroscopy in the chemical sciences, Chem. Soc. Rev., 47 (2018) 2534-2553.

[3] W.R. Hagen, EPR spectroscopy as a probe of metal centres in biological systems, Dalt. Trans., (2006) 4415-4434.

[4] J.P. Klare, Site-directed spin labeling EPR spectroscopy in protein research, Biol. Chem., 394 (2013) 1281-1300.

[5] W.L. Hubbell, H.S. Mchaourab, C. Altenbach, M.A. Lietzow, Watching proteins move using site-directed spin labeling, Structure, 4 (1996) 779-783.

[6] C.S. Klug, J.B. Feix, Methods and Applications of Site-Directed Spin Labeling EPR Spectroscopy, Methods Cell Biol., 84 (2008) 617-658.

[7] G.Z. Sowa, P.Z. Qin, Site directed spin labelling studies on nucleic acid structures and dynamics, , Prog. Nucleic Acid Res. Mol. Biol., 82 (2008) 147-197

[8] W.K. Subczynski, M. Raguz, J. Widomska, Studying lipid organization in biological membranes using liposomes and EPR spin labeling, Methods Mol. Biol., 606 (2010) 247269.

[9] D. Goldfarb, Gd3+ spin labeling for distance measurements by pulse EPR spectroscopy, Phys. Chem. Chem. Phys., 16 (2014) 9685-9699.

[10] Y. Yang, Y.J. Gong, A. Litvinov, H.K. Liu, F. Yang, X.C. Su, D. Goldfarb, Generic tags for $\mathrm{Mn}(\mathrm{II})$ and Gd(III) spin labels for distance measurements in proteins, Phys. Chem. Chem. Phys., 19 (2017) 26944-26956. 
[11] E. Bordignon, EPR spectroscopy of nitroxide spin probes, EMagRes, 6 (2017) 235-254.

[12] J.J. Jassoy, C.A. Heubach, T. Hett, F. Bernhard, F.R. Haege, G. Hagelueken, O. Schiemann, Site Selective and Efficient Spin Labeling of Proteins with a Maleimide-Functionalized Trityl Radical for Pulsed Dipolar EPR Spectroscopy, Molecules, 24 (2019) 1-18.

[13] W.L. Hubbell, C.J. López, C. Altenbach, Z. Yang, Technological advances in site-directed spin labeling of proteins, Curr. Opin. Struct. Biol., 23 (2013) 725-733.

[14] G. Jeschke, DEER distance measurements on proteins, Annu. Rev. Phys. Chem., 63 (2012) 419-446.

[15] S. Longhi, V. Belle, A. Fournel, B. Guigliarelli, F. Carrière, Probing structural transitions in both structured and disordered proteins using site-directed spin-labeling EPR spectroscopy, J. Pept. Sci., 17 (2011) 315-328.

[16] I.D. Sahu, G.A. Lorigan, Electron paramagnetic resonance as a tool for studying membrane proteins, Biomolecules, 10 (2020) 1-25.

[17] E. Bordignon, Y. Polyhach, EPR techniques to probe insertion and conformation of spinlabeled proteins in lipid bilayers, Methods Mol. Biol., 974 (2013) 329-355.

[18] M. Drescher, No Title, Top. Curr. Chem., 321 (2012) 91-119.

[19] P. Consentius, U. Gohlke, B. Loll, C. Alings, U. Heinemann, M.C. Wahl, T. Risse, Combining EPR spectroscopy and X-ray crystallography to elucidate the structure and dynamics of conformationally constrained spin labels in T4 lysozyme single crystals, Phys. Chem. Chem. Phys., 19 (2017) 20723-20734.

[20] P. Consentius, U. Gohlke, B. Loll, C. Alings, R. Müller, U. Heinemann, M. Kaupp, M. Wahl, T. Risse, Tracking Transient Conformational States of T4 Lysozyme at Room Temperature Combining X-ray Crystallography and Site-Directed Spin Labeling, J. Am. Chem. Soc., 138 (2016) 12868-12875.

[21] H.A. Koteiche, S. Chiu, R.L. Majdoch, P.L. Stewart, H.S. Mchaourab, Atomic models by Cryo-EM and site-directed spin labeling: Application to the N-terminal region of Hsp165, Structure, 13 (2005) 1165-1171.

[22] O.H. Griffith, H.M. McConnell, a Nitroxide-Maleimide Spin Label, Proc. Natl. Acad. Sci., 55 (1966) 8-11.

[23] S.A. Sundberg, W.L. Hubbell, Investigation of surface potential asymmetry in phospholipid vesicles by a spin label relaxation method, Biophys. J., 49 (1986) 553-562.

[24] C. Altenbach, W. Froncisz, J.S. Hyde, W.L. Hubbell, Conformation of spin-labeled melittin at membrane surfaces investigated by pulse saturation recovery and continuous wave power saturation electron paramagnetic resonance, Biophys. J., 56 (1989) 1183-1191. 
[25] I. García-Rubio, EPR of site-directed spin-labeled proteins: A powerful tool to study structural flexibility, Arch. Biochem. Biophys., 684 (2020).

[26] E. Mileo, E. Etienne, M. Martinho, R. Lebrun, V. Roubaud, P. Tordo, B. Gontero, B. Guigliarelli, S.R.A. Marque, V. Belle, Enlarging the panoply of site-directed spin labeling electron paramagnetic resonance (SDSL-EPR): Sensitive and selective spin-labeling of tyrosine using an isoindoline-based nitroxide, Bioconjug. Chem., 24 (2013) 1110-1117.

[27] M.R. Fleissner, E.M. Brustad, T. Kálai, C. Altenbach, D. Cascio, F.B. Peters, K. Hideg, P.G. Schultz, W.L. Hubbell, Site-directed spin labeling of a genetically encoded unnatural amino acid, Proc. Natl. Acad. Sci. U. S. A., 106 (2009) 21637-21642.

[28] M.J. Schmidt, J. Borbas, M. Drescher, D. Summerer, A genetically encoded spin label for electron paramagnetic resonance distance measurements, J. Am. Chem. Soc., 136 (2014) 1238-1241.

[29] A. Kugele, B. Silkenath, J. Langer, V. Wittmann, M. Drescher, Protein Spin Labeling with a Photocaged Nitroxide Using Diels-Alder Chemistry, ChemBioChem, 20 (2019) 2479-2484.

[30] S. Schreier, J.C. Bozelli, N. Marín, R.F.F. Vieira, C.R. Nakaie, The spin label amino acid TOAC and its uses in studies of peptides: Chemical, physicochemical, spectroscopic, and conformational aspects, Biophys. Rev., 4 (2012) 45-66.

[31] S. Stoller, G. Sicoli, T.Y. Baranova, M. Bennati, U. Diederichsen, TOPP: A novel nitroxidelabeled amino acid for EPR distance measurements, Angew. Chemie - Int. Ed., 50 (2011) 9743-9746.

[32] D. Fass, C. Thorpe, Chemistry and Enzymology of Disulfide Cross-Linking in Proteins, Chem. Rev., 118 (2018) 1169-1198.

[33] A. Clark, J. Sedhom, H. Elajaili, G.R. Eaton, S.S. Eaton, Dependence of electron paramagnetic resonance spectral lineshapes on molecular tumbling: Nitroxide radical in water:glycerol mixtures, Concepts Magn. Reson. Part A Bridg. Educ. Res., 45A (2016).

[34] H.J. Steinhoff, A simple method for determination of rotational correlation times and separation of rotational and polarity effects from EPR spectra of spin-labeled biomolecules in a wide correlation time range, J. Biochem. Biophys. Methods, 17 (1988) 237-247.

[35] H.S. Mchaourab, M.A. Lietzow, K. Hideg, W.L. Hubbell, Motion of spin-labeled side chains in T4 lysozyme Correlation with protein structure and dynamics, Biochemistry, 35 (1996) 7692-7704.

[36] S. Stoll, A. Schweiger, EasySpin, a comprehensive software package for spectral simulation and analysis in EPR, J. Magn. Reson., 178 (2006) 42-55.

[37] E. Etienne, N. Le Breton, M. Martinho, E. Mileo, V. Belle, SimLabel: a graphical user 
interface to simulate continuous wave EPR spectra from site-directed spin labeling experiments, Magn. Reson. Chem., 55 (2017) 714-719.

[38] C. Altenbach, C.J. López, K. Hideg, W.L. Hubbell, Exploring Structure, Dynamics, and Topology of Nitroxide Spin-Labeled Proteins Using Continuous-Wave Electron Paramagnetic Resonance Spectroscopy, Methods Enzymol., 564 (2015) 59-100.

[39] A. Kavalenka, I. Urbančič, V. Belle, S. Rouger, S. Costanzo, S. Kure, A. Fournel, S. Longhi, B. Guigliarelli, J. Strancar, Conformational analysis of the partially disordered measles virus N TAIL-XD complex by SDSL EPR spectroscopy, Biophys. J., 98 (2010) 1055-1064.

[40] T.I. Smirnova, A.I. Smirnov, Peptide-Membrane Interactions by Spin-Labeling EPR, 1st ed., Elsevier Inc., 2015.

[41] H. Sameach, S. Ruthstein, EPR Distance Measurements as a Tool to Characterize ProteinDNA Interactions, Isr. J. Chem., 59 (2019) 980-989.

[42] M. Palombo, A. Bonucci, E. Etienne, S. Ciurli, V.N. Uversky, B. Guigliarelli, V. Belle, E. Mileo, B. Zambelli, The relationship between folding and activity in UreG, an intrinsically disordered enzyme, Sci. Rep., 7 (2017).

[43] M. Sarewicz, S. Szytuła, M. Dutka, A. Osyczka, W. Froncisz, Estimation of binding parameters for the protein-protein interaction using a site-directed spin labeling and EPR spectroscopy, Eur. Biophys. J., 37 (2008) 483-493.

[44] A.A. Malygin, O.A. Krumkacheva, D.M. Graifer, I.O. Timofeev, A.S. Ochkasova, M.I. Meschaninova, A.G. Venyaminova, M. V. Fedin, M. Bowman, G.G. Karpova, E.G. Bagryanskaya, Exploring the interactions of short RNAs with the human 40S ribosomal subunit near the mRNA entry site by EPR spectroscopy, Nucleic Acids Res., 47 (2019) $11850-11860$.

[45] O. Duss, M. Yulikov, F.H.T. Allain, G. Jeschke, Combining NMR and EPR to determine structures of large RNAs and protein-RNA complexes in solution, Methods Enzymol., 558 (2015) 279-331.

[46] A. Bonucci, E. Caldaroni, E. Balducci, R. Pogni, A Spectroscopic Study of the Aggregation State of the Human Antimicrobial Peptide LL-37 in Bacterial versus Host Cell Model Membranes, Biochemistry, 54 (2015).

[47] A.D.J. Freeman, M. Stevens, A.C. Declais, A. Leahy, K. Mackay, H. El Mkami, D.M.J. Lilley, D.G. Norman, Analysis of the Intrinsically Disordered N-Terminus of the DNA Junction-Resolving Enzyme T7 Endonuclease I: Identification of Structure Formed upon DNA Binding, Biochemistry, 55 (2016) 4166-4172.

[48] N. Le Breton, S. Longhi, A. Rockenbauer, B. Guigliarelli, S.R.A. Marque, V. Belle, M. 
Martinho, Probing the dynamic properties of two sites simultaneously in a protein-protein interaction process: A SDSL-EPR study, Phys. Chem. Chem. Phys., 21 (2019) 22584-22588.

[49] C. Altenbach, W. Froncisz, R. Hemker, H. Mchaourab, W.L. Hubbell, Accessibility of nitroxide side chains: Absolute Heisenberg exchange rates from power saturation EPR, Biophys. J., 89 (2005) 2103-2112.

[50] J. Pyka, J. Ilnicki, C. Altenbach, W.L. Hubbell, W. Froncisz, Accessibility and dynamics of nitroxide side chains in T4 lysozyme measured by saturation recovery EPR, Biophys. J., 89 (2005) 2059-2068.

[51] K.J. Oh, P. Singh, K. Lee, K. Foss, S. Lee, M. Park, S. Lee, S. Aluvila, M. Park, P. Singh, R.S. Kim, J. Symersky, D.E. Walters, Conformational changes in BAK, a pore-forming proapoptotic Bcl-2 family member, upon membrane insertion and direct evidence for the existence of BH3-BH3 contact interface in BAK homo-oligomers, J. Biol. Chem., 285 (2010) $28924-28937$.

[52] G. Jeschke, Y. Polyhach, Distance measurements on spin-labelled biomacromolecules by pulsed electron paramagnetic resonance, Phys. Chem. Chem. Phys., 9 (2007) 1895-1910.

[53] D. Goldfarb, Pulse EPR in biological systems - Beyond the expert's courtyard, J. Magn. Reson., 306 (2019) 102-108.

[54] T. Schmidt, M.A. Wälti, J.L. Baber, E.J. Hustedt, G.M. Clore, Long Distance Measurements up to $160 \AA$ in the GroEL Tetradecamer Using Q-Band DEER EPR Spectroscopy, Angew. Chemie, 128 (2016) 16137-16141.

[55] S. Milikisyants, F. Scarpelli, M.G. Finiguerra, M. Ubbink, M. Huber, A pulsed EPR method to determine distances between paramagnetic centers with strong spectral anisotropy and radicals: The dead-time free RIDME sequence, J. Magn. Reson., 201 (2009) 48-56.

[56] P. Schöps, P.E. Spindler, A. Marko, T.F. Prisner, Broadband spin echoes and broadband SIFTER in EPR, J. Magn. Reson., 250 (2015) 55-62.

[57] T.H. Edwards, S. Stoll, Optimal Tikhonov regularization for DEER spectroscopy, J. Magn. Reson., 288 (2018) 58-68.

[58] Y.W. Chiang, P.P. Borbat, J.H. Freed, The determination of pair distance distributions by pulsed ESR using Tikhonov regularization, J. Magn. Reson., 172 (2005) 279-295.

[59] Y. Polyhach, E. Bordignon, R. Tschaggelar, S. Gandra, A. Godt, G. Jeschke, High sensitivity and versatility of the DEER experiment on nitroxide radical pairs at Q-band frequencies, Phys. Chem. Chem. Phys., 14 (2012) 10762-10773.

[60] I.M.S. de Vera, M.E. Blackburn, L. Galiano, G.E. Fanucci, Pulsed EPR distance measurements in soluble proteins by site-directed spin labeling (SDSL), Curr. Protoc. Protein 
Sci., 2013 (2013) 17.17.1-17.17.29.

[61] V. Meyer, M.A. Swanson, L.J. Clouston, P.J. Boratyński, R.A. Stein, H.S. McHaourab, A. Rajca, S.S. Eaton, G.R. Eaton, Room-temperature distance measurements of immobilized Spin-labeled Protein by DEER/PELDOR, Biophys. J., 108 (2015) 1213-1219.

[62] Yulikov M., Spectroscopically orthogonal spin labels and distance measurements in biomolecules, Electron Paramagn. Reson., 24 (2014).

[63] Z. Wu, A. Feintuch, A. Collauto, L.A. Adams, L. Aurelio, B. Graham, G. Otting, D. Goldfarb, Selective Distance Measurements Using Triple Spin Labeling with Gd3+, Mn2+, and a Nitroxide, J. Phys. Chem. Lett., 8 (2017) 5277-5282.

[64] C. Gmeiner, D. Klose, E. Mileo, V. Belle, S.R.A. Marque, G. Dorn, F.H.T. Allain, B. Guigliarelli, G. Jeschke, M. Yulikov, Orthogonal tyrosine and cysteine site-directed spin labeling for dipolar pulse EPR spectroscopy on proteins, J. Phys. Chem. Lett., 8 (2017) 4852-4857.

[65] L. Garbuio, E. Bordignon, E.K. Brooks, W.L. Hubbell, G. Jeschke, M. Yulikov, Orthogonal spin labeling and $\mathrm{Gd}(\mathrm{III})$-nitroxide distance measurements on bacteriophage T4-lysozyme, J. Phys. Chem. B, 117 (2013) 3145-3153.

[66] I. Ritsch, H. Hintz, G. Jeschke, A. Godt, M. Yulikov, Improving the accuracy of Cu(ii)nitroxide RIDME in the presence of orientation correlation in water-soluble $\mathrm{Cu}$ (ii)-nitroxide rulers, Phys. Chem. Chem. Phys., 21 (2019) 9810-9830.

[67] L. V. Kulik, S.A. Dzuba, I.A. Grigoryev, Y.D. Tsvetkov, Electron dipole-dipole interaction in ESEEM of nitroxide biradicals, Chem. Phys. Lett., 343 (2001) 315-324.

[68] D.P. Claxton, K.L. Jagessar, P. Ryan Steed, R.A. Stein, H.S. Mchaourab, Sodium and proton coupling in the conformational cycle of a MATE antiporter from Vibrio cholerae, Proc. Natl. Acad. Sci. U. S. A., 115 (2018) E6182-E6190.

[69] J.M. Plitzko, B. Schuler, P. Selenko, Structural Biology outside the box — inside the cell, Curr. Opin. Struct. Biol., 46 (2017) 110-121.

[70] R.J. Ellis, Macromolecular crowding: An important but neglected aspect of the intracellular environment, Curr. Opin. Struct. Biol., 11 (2001) 114-119.

[71] I.M. Kuznetsova, K.K. Turoverov, V.N. Uversky, What macromolecular crowding can do to a protein, 2014.

[72] F.X. Theillet, A. Binolfi, T. Frembgen-Kesner, K. Hingorani, M. Sarkar, C. Kyne, C. Li, P.B. Crowley, L. Gierasch, G.J. Pielak, A.H. Elcock, A. Gershenson, P. Selenko, Physicochemical properties of cells and their effects on intrinsically disordered proteins (IDPs), Chem. Rev., 114 (2014) 6661-6714. 
[73] E. Luchinat, L. Banci, A unique tool for cellular structural biology: In-cell NMR, J. Biol. Chem., 291 (2016) 3776-3784.

[74] E. Luchinat, L. Banci, In-cell NMR: A topical review, IUCrJ, 4 (2017) 108-118.

[75] P. Selenko, G. Wagner, Looking into live cells with in-cell NMR spectroscopy, J. Struct. Biol., 158 (2007) 244-253.

[76] K. Okamoto, Y. Sako, Recent advances in FRET for the study of protein interactions and dynamics, Curr. Opin. Struct. Biol., 46 (2017) 16-23.

[77] Y. Chen, J.D. Mills, A. Periasamy, Protein localization in living cells and tissues using FRET and FLIM, Differentiation, 71 (2003) 528-541.

[78] A. Bonucci, O. Ouari, B. Guigliarelli, V. Belle, E. Mileo, In-Cell EPR: Progress towards Structural Studies Inside Cells, ChemBioChem, 21 (2020) 451-460.

[79] I. Krstić, R. Hänsel, O. Romainczyk, J.W. Engels, V. Dötsch, T.F. Prisner, Long-range distance measurements on nucleic acids in cells by pulsed EPR spectroscopy, Angew. Chemie - Int. Ed., 50 (2011) 5070-5074.

[80] M. Azarkh, O. Okle, V. Singh, I.T. Seemann, J.S. Hartig, D.R. Dietrich, M. Drescher, Longrange distance determination in a DNA model system inside Xenopus laevis Oocytes by InCell Spin-Label EPR, ChemBioChem, 12 (2011) 1992-1995.

[81] A.A. Bobko, I.A. Kirilyuk, I.A. Grigor'ev, J.L. Zweier, V. V. Khramtsov, Reversible reduction of nitroxides to hydroxylamines: Roles for ascorbate and glutathione, Free Radic. Biol. Med., 42 (2007) 404-412.

[82] K. Chen, H.M. Swartz, Oxidation of hydroxylamines to nitroxide spin labels in living cells Kai Chen and Harold M Swartz, Tempo, 970 (1988) 270-277.

[83] M. Azarkh, O. Okle, P. Eyring, D.R. Dietrich, M. Drescher, Evaluation of spin labels for incell EPR by analysis of nitroxide reduction in cell extract of Xenopus laevis oocytes, J. Magn. Reson., 212 (2011) 450-454.

[84] M.J. Schmidt, A. Fedoseev, D. Summerer, M. Drescher, Genetically Encoded Spin Labels for in Vitro and In-Cell EPR Studies of Native Proteins, 1st ed., Elsevier Inc., 2015.

[85] A.P. Jagtap, I. Krstic, N.C. Kunjir, R. Hänsel, T.F. Prisner, S.T. Sigurdsson, Sterically shielded spin labels for in-cell EPR spectroscopy: Analysis of stability in reducing environment, Free Radic. Res., 49 (2015) 78-85.

[86] J.T. Paletta, M. Pink, B. Foley, S. Rajca, A. Rajca, Synthesis and reduction kinetics of sterically shielded pyrrolidine nitroxides, Org. Lett., 14 (2012) 5322-5325.

[87] M.M. Haugland, J.E. Lovett, E.A. Anderson, Advances in the synthesis of nitroxide radicals for use in biomolecule spin labelling, Chem. Soc. Rev., 47 (2018) 668-680. 
[88] S. Bleicken, T.E. Assafa, H. Zhang, C. Elsner, I. Ritsch, M. Pink, S. Rajca, G. Jeschke, A. Rajca, E. Bordignon, gem-Diethyl Pyrroline Nitroxide Spin Labels: Synthesis, EPR Characterization, Rotamer Libraries and Biocompatibility, ChemistryOpen, (2019) 10571065.

[89] G. Karthikeyan, A. Bonucci, G. Casano, G. Gerbaud, S. Abel, V. Thomé, L. Kodjabachian, A. Magalon, B. Guigliarelli, V. Belle, O. Ouari, E. Mileo, A Bioresistant Nitroxide Spin Label for In-Cell EPR Spectroscopy: In Vitro and In Oocytes Protein Structural Dynamics Studies, Angew. Chemie - Int. Ed., 57 (2018).

[90] H.E. Chau C., Actis P., Methods for protein delivery into cells: from current approaches to future prospectives, Biochem. Soc. Trans., 48 (2020) 357-365.

[91] B. Joseph, A. Sikora, D.S. Cafiso, Ligand Induced Conformational Changes of a Membrane Transporter in E coli Cells Observed with DEER/PELDOR, J. Am. Chem. Soc., 138 (2016) 1844-1847.

[92] S. Dunkel, L.P. Pulagam, H.J. Steinhoff, J.P. Klare, In vivo EPR on spin labeled colicin A reveals an oligomeric assembly of the pore-forming domain in E coli membranes, Phys. Chem. Chem. Phys., 17 (2015) 4875-4878.

[93] Y. Yang, F. Yang, Y.J. Gong, T. Bahrenberg, A. Feintuch, X.C. Su, D. Goldfarb, High Sensitivity In-Cell EPR Distance Measurements on Proteins using an Optimized Gd(III) Spin Label, J. Phys. Chem. Lett., 9 (2018) 6119-6123.

[94] Y. Yang, B. Bin Pan, X. Tan, F. Yang, Y. Liu, X.C. Su, D. Goldfarb, In-Cell Trityl-Trityl Distance Measurements on Proteins, J. Phys. Chem. Lett., 11 (2020) 1141-1147. 


\section{Figures}
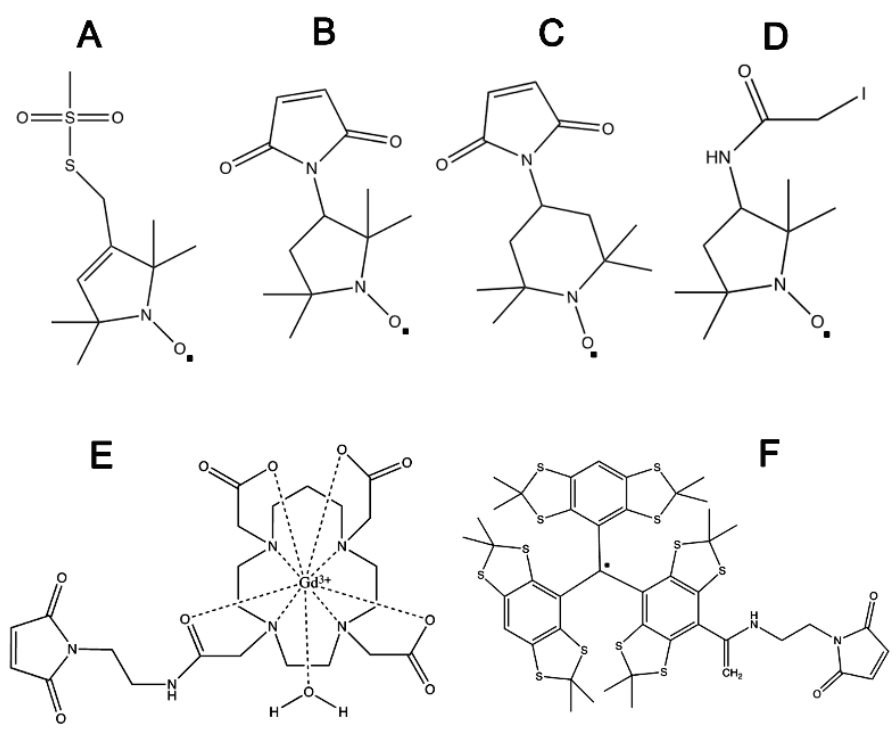

Fig.1: Chemical structures of various spin labels used for SDSL-EPR spectroscopy: A) (S-(1-oxyl-2,2,5,5tetramethyl-2,5-dihydro-1H-pyrrol-3-yl)methyl methanethiosulfonate spin label (MTSL); B) 3-Maleimido2,2,5,5-tetramethyl-1-pyrrolidinyloxy free radical (MA-PX); C) 4-Maleimido-2,2,6,6-tetramethyl-1piperidinyloxy free radical (4-maleimido TEMPO); D) 3-(2-Iodoacetamido)-2,2,5,5-tetramethyl-1pyrrolidinyloxy free radical (Iodoacetamide-proxyl); E) 1,4,7,10- tetraazacyclododecane-1,4,7-tris-acetic acid-10-maleimidoethylacetamide- Gd(III) (Gd(III)-DOTA spin label) ; F) Maleimido functionalized tritylradical spin label. 

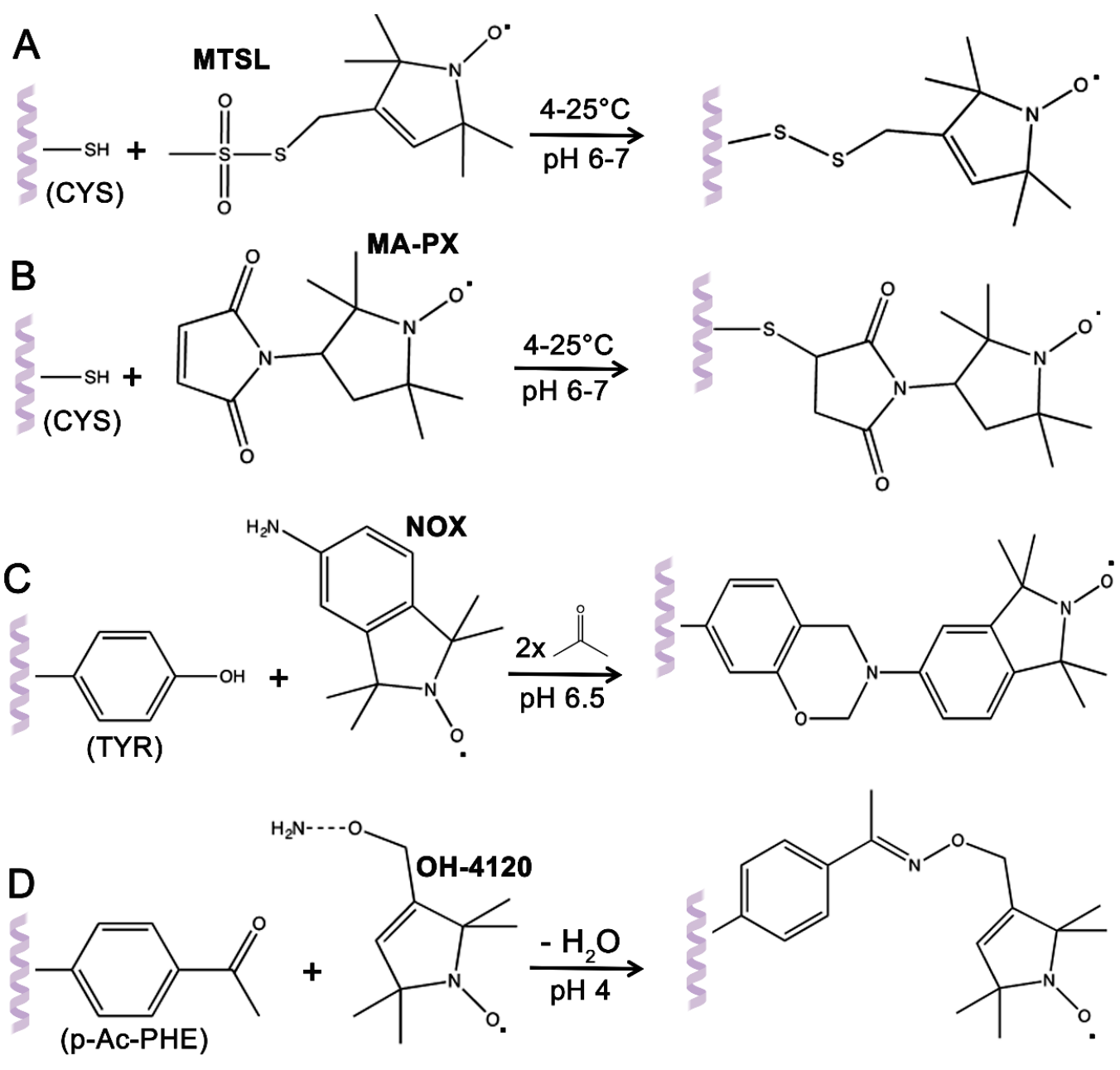

Fig. 2: Different types of SDSL reactions: A-B) SDSL reaction to target cysteine using MTSL and 3maleimido-Proxyl (MA-PX) nitroxide labels; C) SDSL reaction on tyrosine using NOX label through a Mannich-type reaction; D) SDSL reaction on the unnatural amino acid p-acetyl-phenylalanine with HO-4120 nitroxide label. 


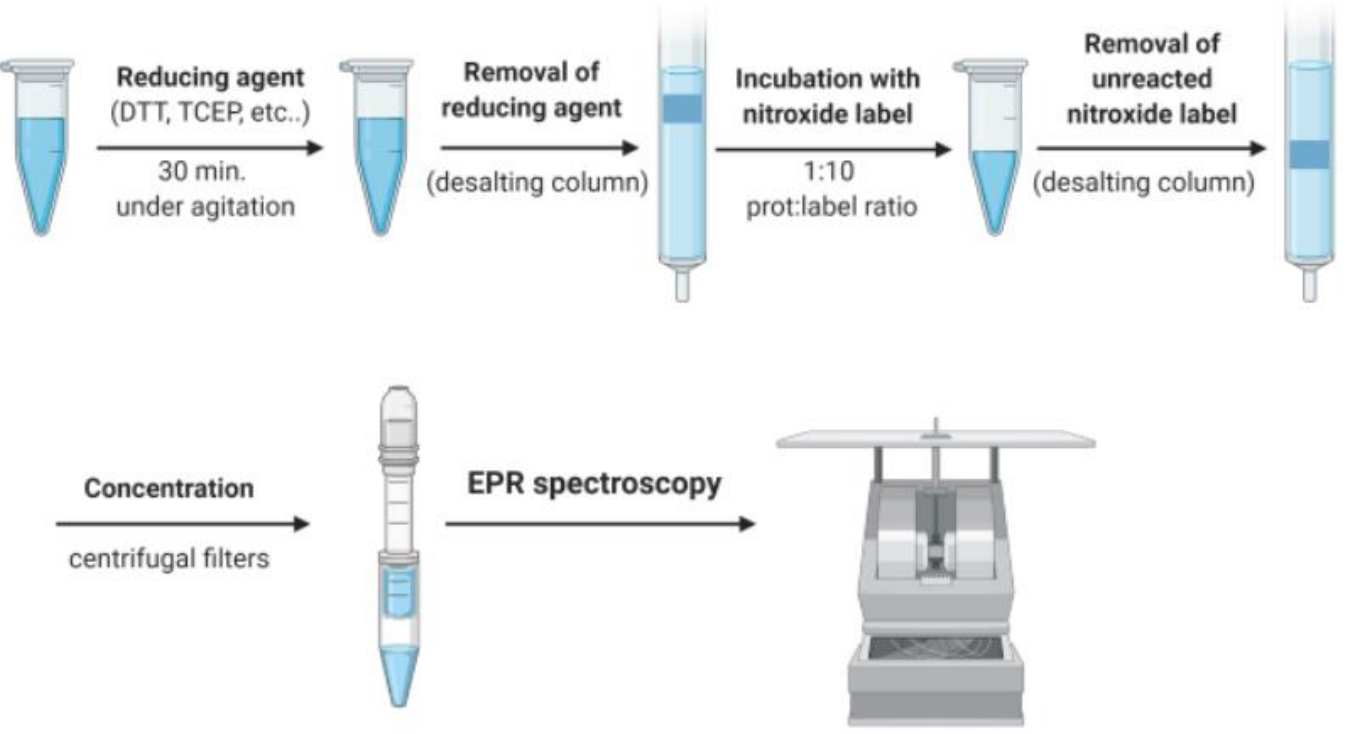

Fig.3: General protocol for standard sample preparation for SDSL-EPR spectroscopy: Scheme of the general protocol for the preparation of spin labelled proteins with nitroxide probes. Initially the protein is incubated for about 30 minutes with a reducing agent. Once the reducing agent is removed by chromatography, the protein is incubated with a ten-fold molar excess of nitroxide spin label and the reaction is maintained under agitation for 1-4 hours. At the end, the unreacted fraction of nitroxide label is removed through chromatography. The nitroxide labelled proteins is obtained in order to be studied by cw- EPR/DEER spectroscopies (image created with Biorender).
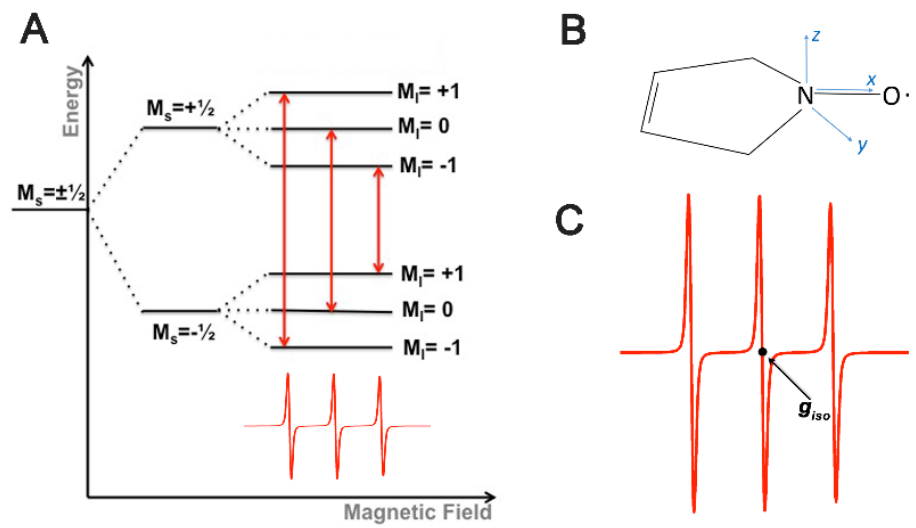

Fig. 4: EPR properties of the nitroxide-based spin labels: A) Energy levels diagram of a nitroxide. The hyperfine interaction of the unpaired electron with the nitrogen nucleus causes the splitting of the electron states, producing three possible transitions (red arrows) corresponding to three spectral lines. B) Axis system of the nitroxide moiety, where the $\mathrm{z}$-axis is oriented parallel to the $p z$ orbital of the nitrogen atom and the $\mathrm{x}$-axis is parallel to the $\mathrm{N}-\mathrm{O}$ bond. C) Typical isotropic EPR spectrum of a free nitroxide label with giso corresponding to the central line. 


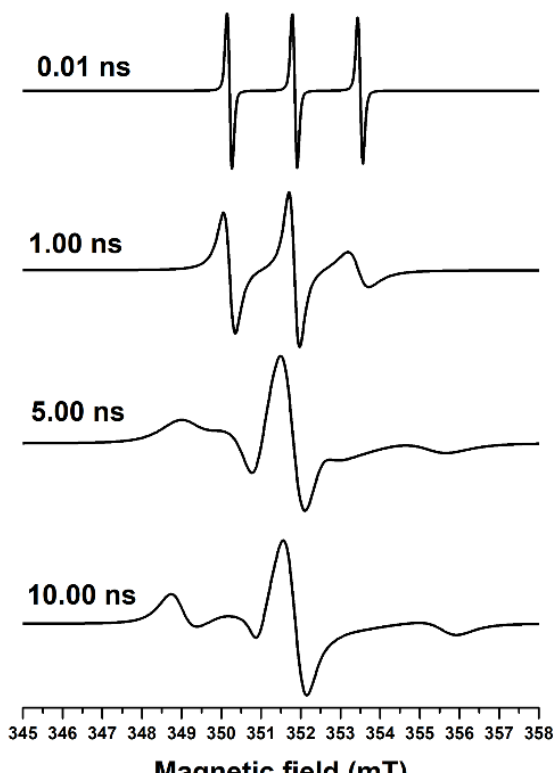

Magnetic field (mT)

Fig. 5: Different EPR spectra of nitroxide-labelled proteins: Simulated X-band EPR (Simlabel software, a graphical user interface of EasySpin [38]) spectra of a nitroxide with different values of $\tau \mathrm{c}$ from fast $(0.01 \mathrm{~ns})$ to slow motion $(10 \mathrm{~ns})$. (The intensities of the simulated spectra have been normalized). 


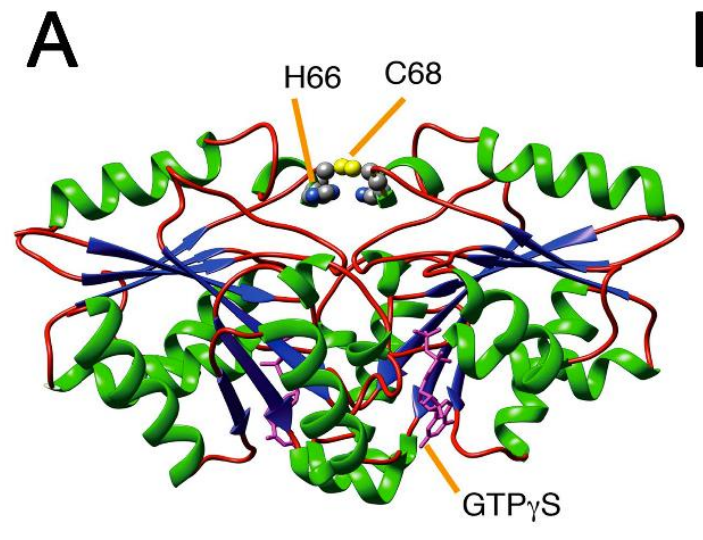

C
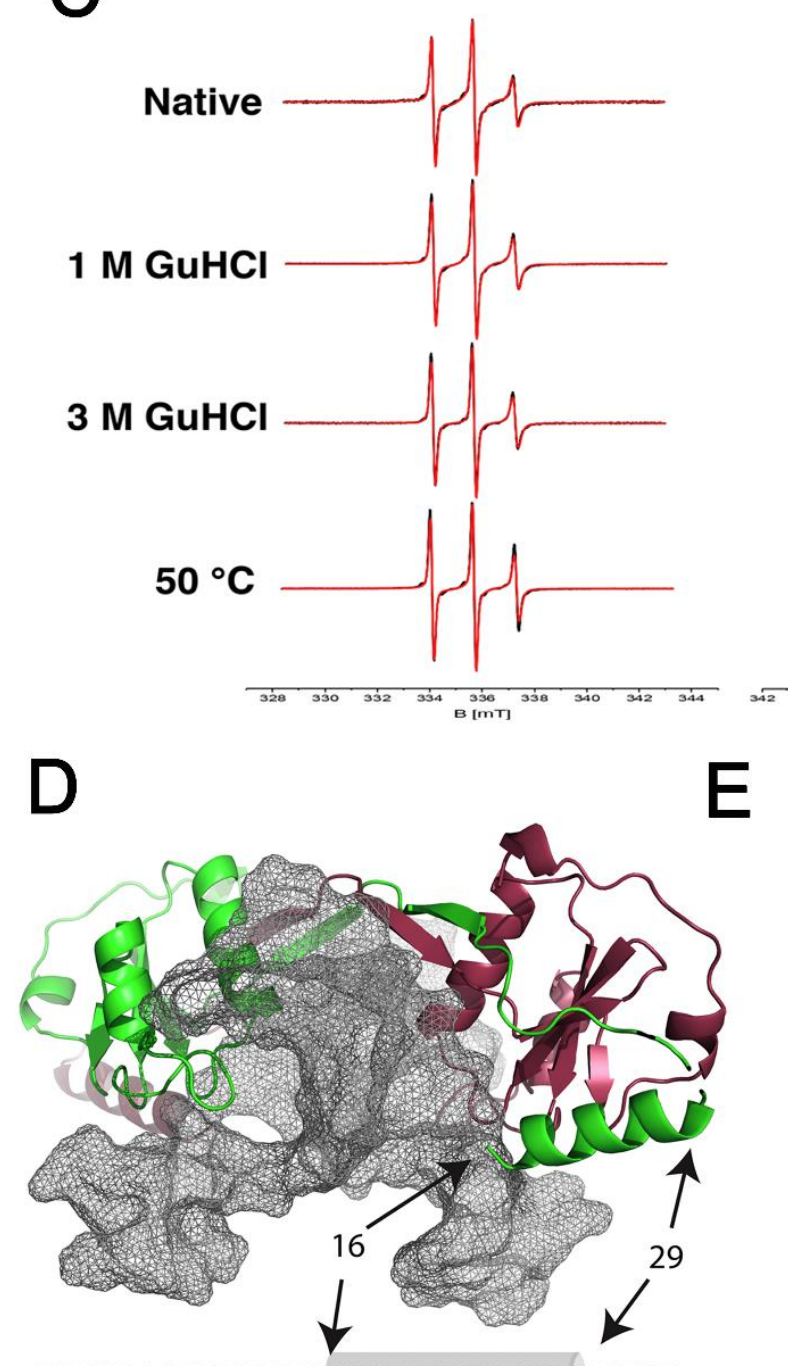

MAGYGAKGIRKVGAFRSGLEDKVSKQLESKGIKFE
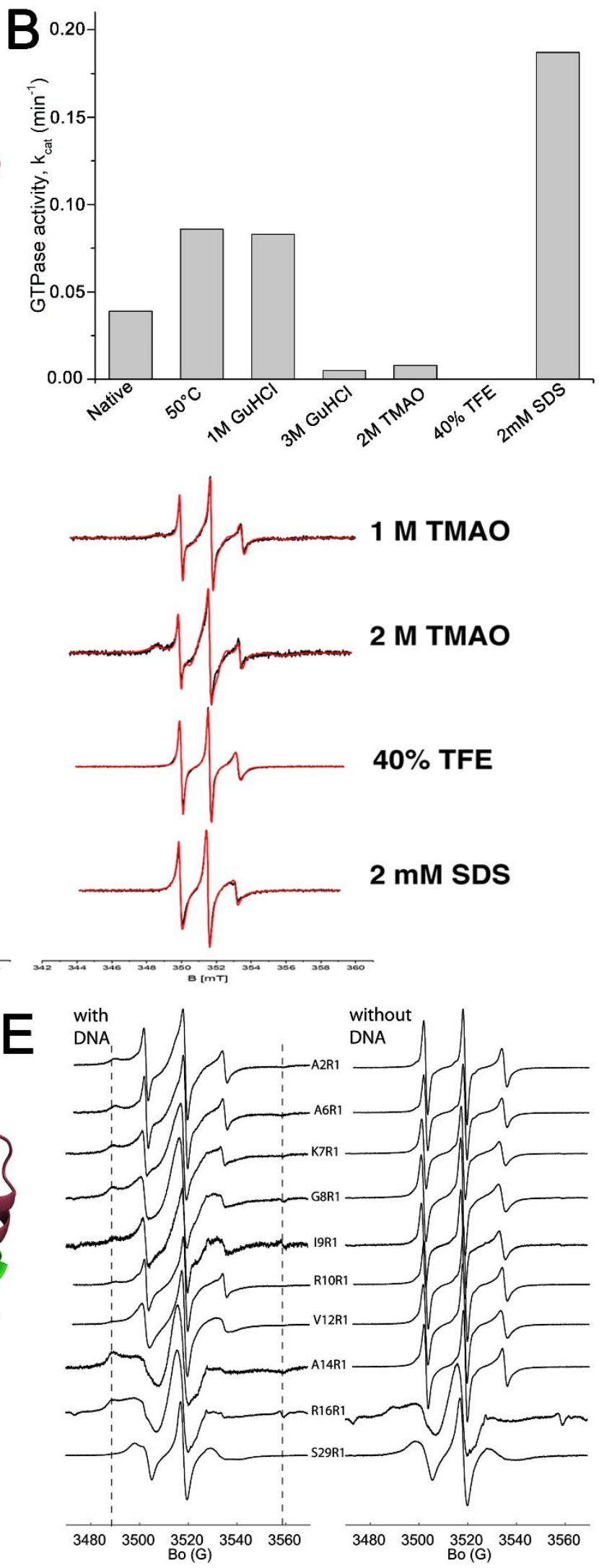

Fig. 6: Examples of two different cw-EPR studies performed with nitroxide labelled proteins: A) Structural representation of the fully folded functional state of of $S p$ - UreG with the metal binding site (H66 and C68) and the GTP $\gamma$ S binding region indicated with orange lines B) GTPase activity 
(kcat) measured for $\mathrm{Sp}$-UreG in the absence (native) and in the presence of different additives C) Superimposed experimental (black) and simulated (red) X-band cw-EPR spectra of MTSL labelled $\mathrm{Sp}$ - UreG on C68 in the absence and in the presence of various additives. The spectral simulations were performed with SimLabel (graphical user interface of Easyspin) D) Structure of endonuclease I (red and green represent a monomer respectively) with DNA junction (grey surface). The undetermined structure of the N-terminal domain corresponds to residues 1-16, the first helix from residues 16-29 is shown in grey on the primary sequence and the nitroxide labelling sites are highlighted. E) X-band cw-EPR spectra of the N-term domain of endonuclease 1 labelled with MTSL at positions 2,6-10,12,14, 16 and 29 in the presence (left) and in the absence (right) of DNA (field position at $3489 \mathrm{G}$ and $3559 \mathrm{G}$ were reported as dotted lines). The figure $\mathrm{A} \& \mathrm{C}$ were adapted from ref [42], while Figure B was created using the data available from the same reference. The Figures C \& D were adapted from ref. [47] link: https://pubs.acs.org/doi/full/https://doi.org/10.1021/acs.bioche m.6b00242). Copyright (2016) American chemical society; future reuses of these images must be directed to the ACS)
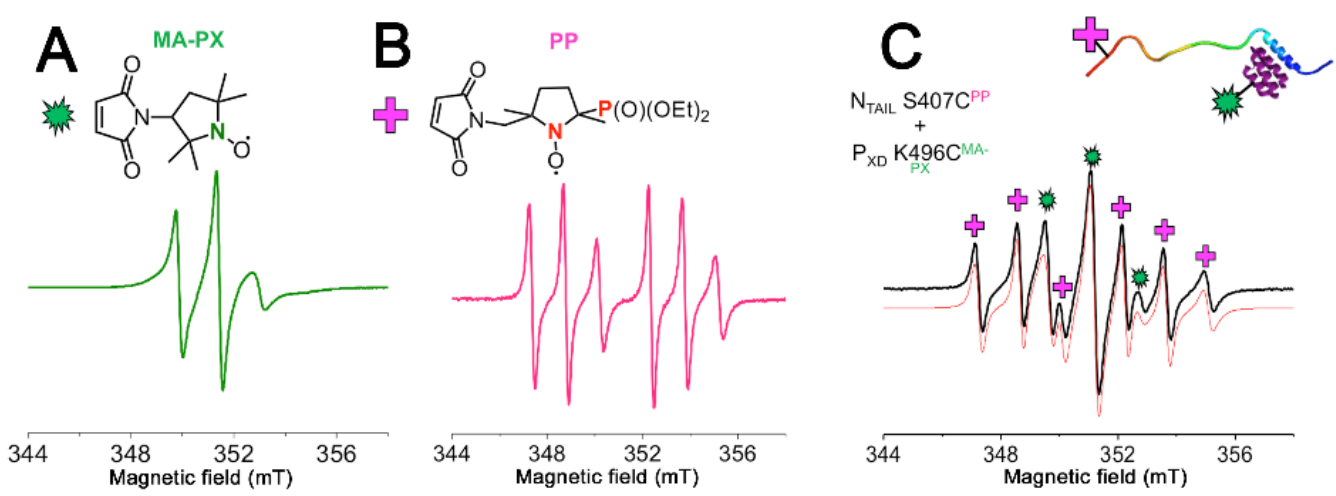

Fig. 7: Example of a cw-EPR study performed with nitroxide labelled proteins: A) Chemical structures of MA-PX and X-band cw-EPR spectrum of PXD K496C MA-PX alone in solution; B) Chemical structures of PP and X-band cw-EPR spectrum of Ntail S470CPP alone in solution; C) Xband cw-ERP spectrum of the equimolar mixture (1:1 ratio) of Ntail S470CPP and PXD K496C MAPX. In the upper panel, the representation of the Ntail (rainbow) - PXD (purple) complex with nitroxide labels attached (MA-PX as "green star" and PP as "pink cross") is reported (see ref [48]). 

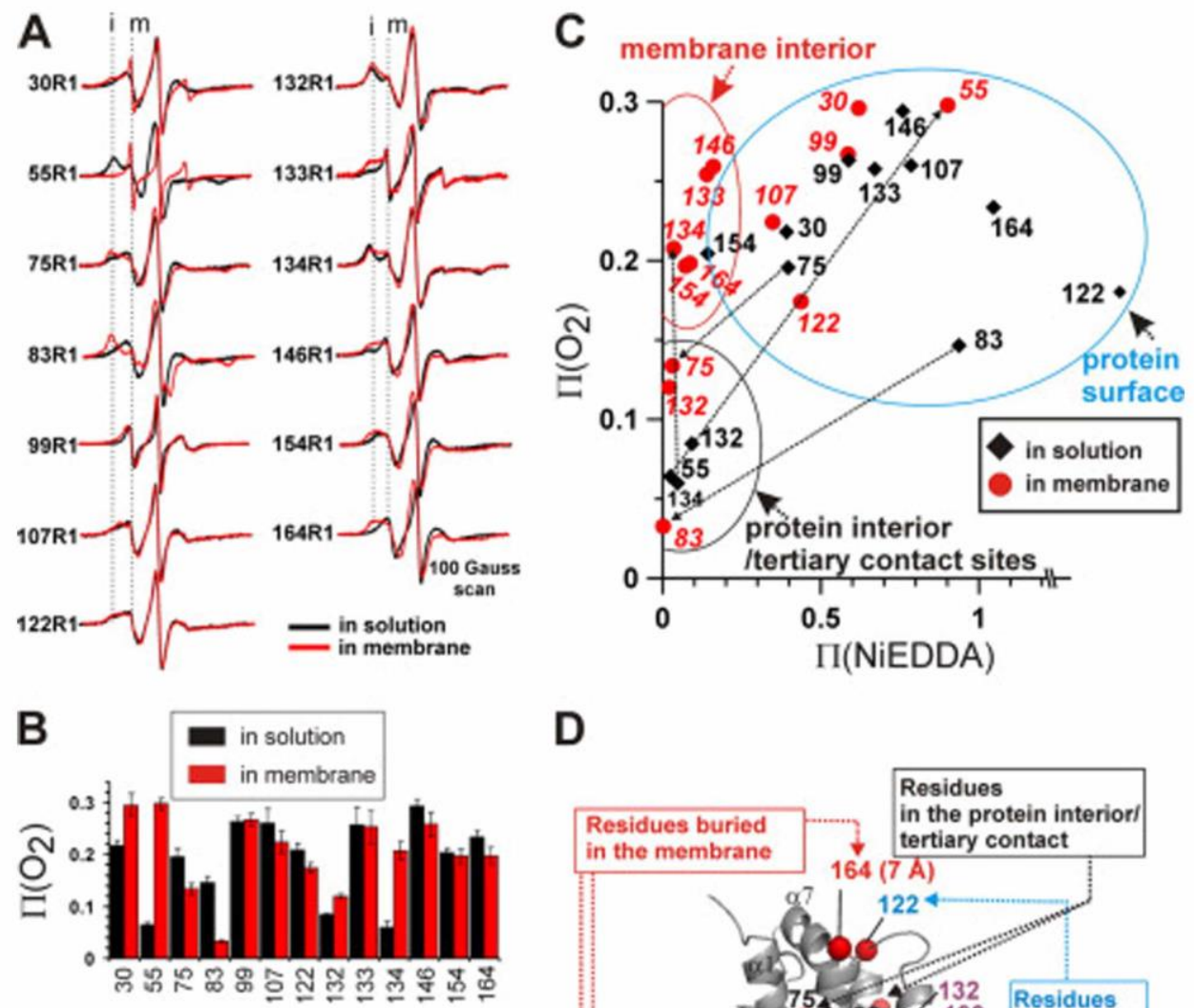

D
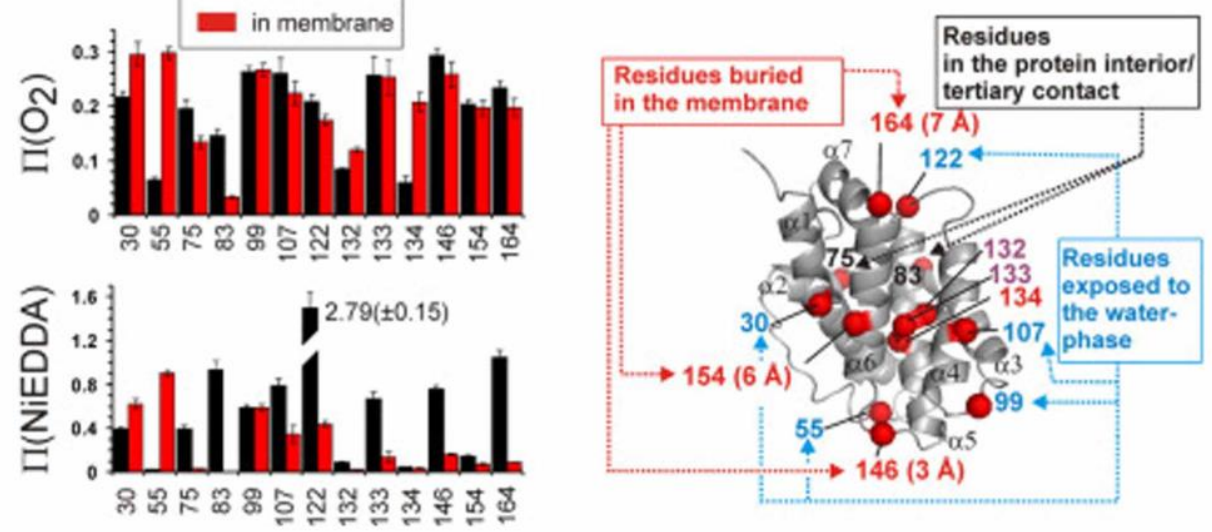

Fig 8: A) EPR spectra of spin labelled BAK variants in solution (black) and in membrane (red). B) accessibility parameters of spin label grafted onto BAK of oxygen $\left(\Pi\left(\mathrm{O}_{2}\right)\right)$ and NiEDDA (П(NiEDDA)) derived from EPR power saturation experiments in solution (black) and in membrane (red). C) Two dimensional plot of $\Pi\left(\mathrm{O}_{2}\right)$ versus $\Pi(\mathrm{NiEDDA})$. The BAK labelling sites are grouped depending on their localization in protein structure. The variation of accessibility parameters from solution to membrane inserted BAK are reported as dotted lines for some labelling positions. D) Homology model of mouse BAK (generated from PDB:2F16)with topological location of labelling sites in the membrane-inserted state. 


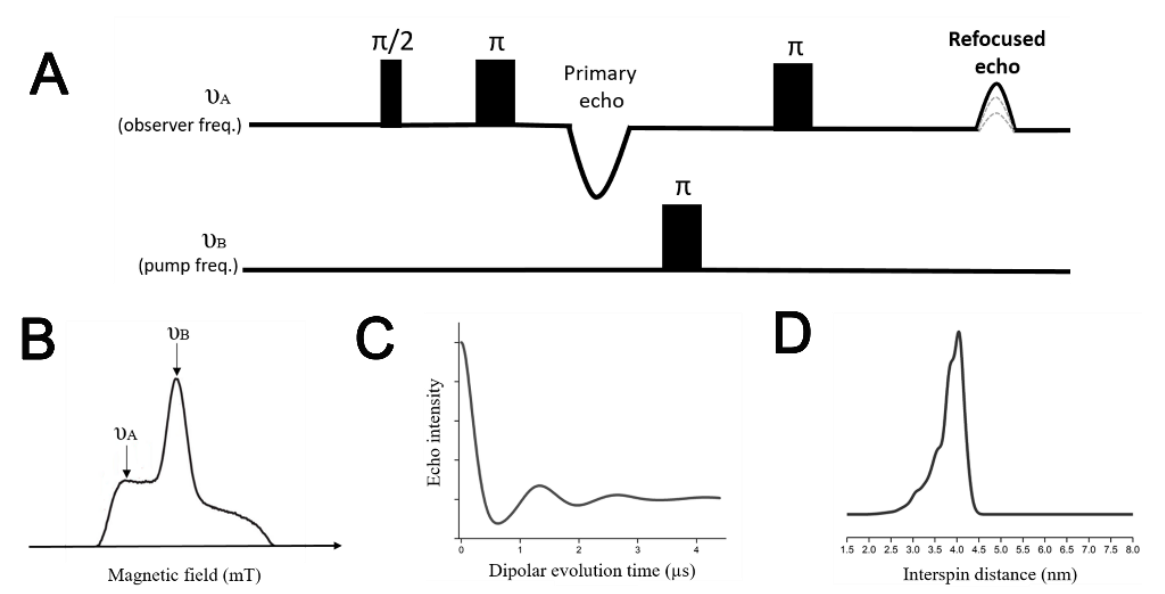

Fig. 9: DEER theory for nitroxide-based spin labels: A) 4-pulses DEER sequence. B) X-band Echofield sweep EPR spectrum of nitroxide labels with the selected observer (vB) and pump (vB) frequencies respectively indicated with arrows. C) Background corrected DEER trace. D) Inter-label distance distribution obtained from the DEER trace reported in the panel B, using DeerAnalysis2019 program. 

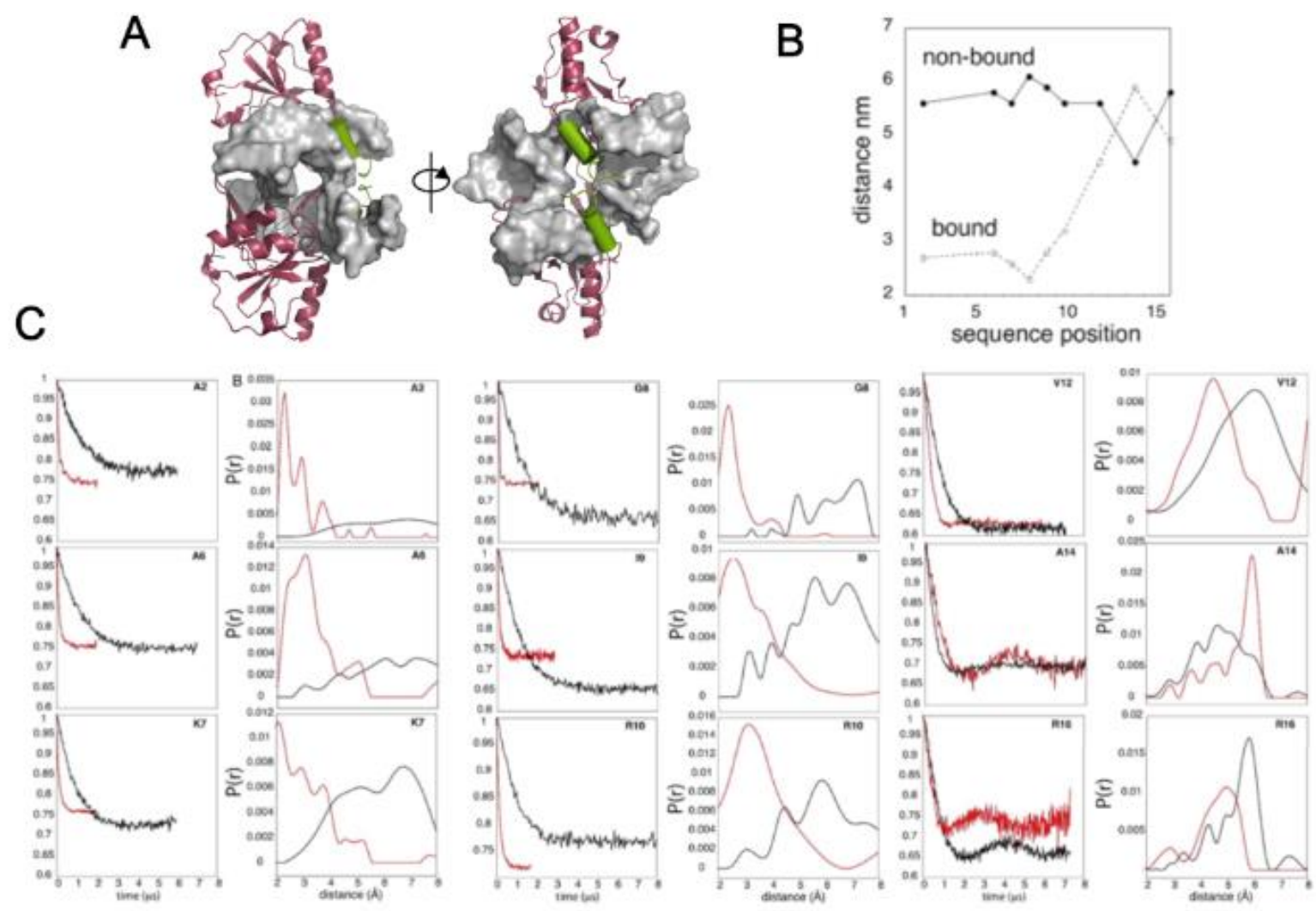

Fig. 10: Example of DEER study performed with a nitroxide labelled protein: A) Two views of endonuclease I structure (magenta from PDB:2PFJ while $\mathrm{N}$-terminal region is represented in green) bound to DNA (grey). B) Plot of the distances derived from DEER experiments as function of the labelling positions in the sequence for endonuclease I in the absence (black) and in the presence (grey) of DNA. C) DEER traces and related distance distributions calculated using Tikhonov regularization for different MTSL labelled mutants of endonuclease I in the absence (black) and in presence (red) of DNA. (Figures were adapted from ref. [47] link: https://pubs. acs.org/doi/full/10.1021/acs.biochem.6b00242). Copyright (2016) American chemical society; future reuses of these images must be directed to the ACS). 

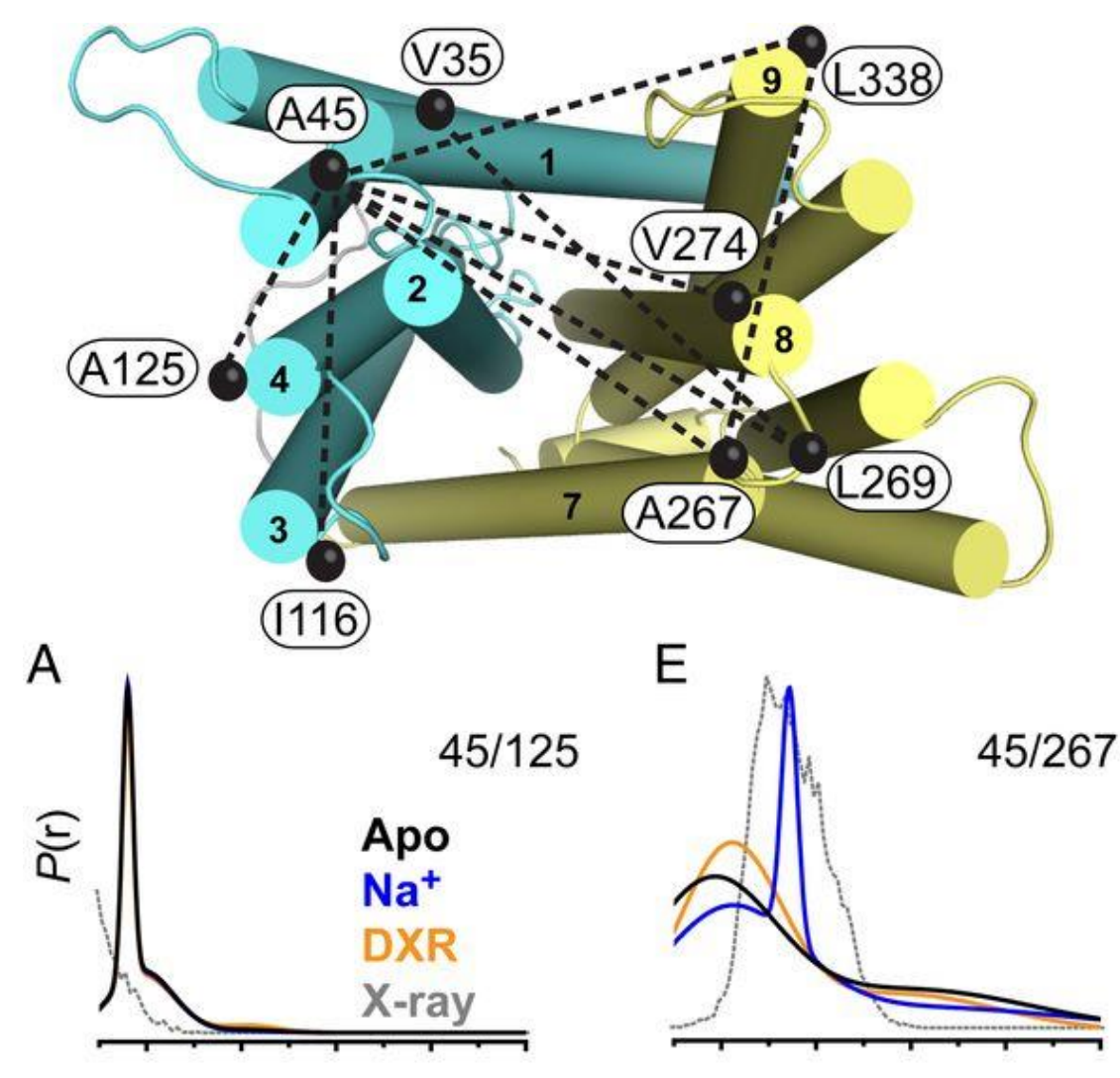

B
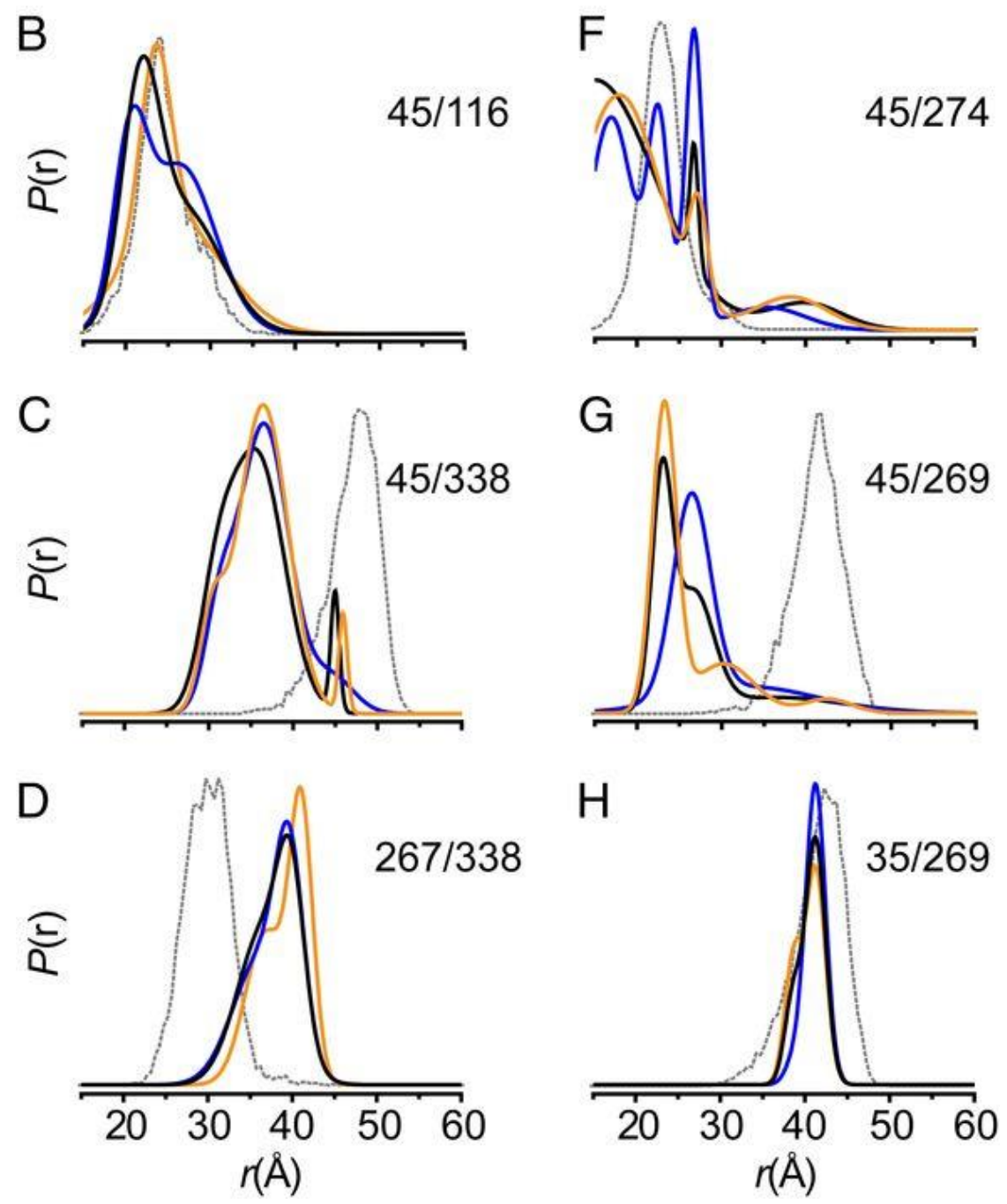
Fig.11: Example of a DEER study performed with a nitroxide labelled protein: (Upper panel) Liganddependent conformational dynamics on the periplasmic side of NorM-Vc; spin label pairs designed to measure distances within the NTD (cyan), CTD (yellow), or between the domains from TM are shown as black spheres connected with dashed lines. (Lower panel) (A-H) DEER distance distributions are shown for the apo-form (black) and in the presence of $\mathrm{Na}+$ (blue) or DXR (orange). The predicted distance distributions derived from X-ray structure (PDB:3MKU) are shown as dotted grey lines. (Figure was taken from ref. [68]: Mchaourab H.S. et al., PNAS, 115 (2018), 6182-6190).
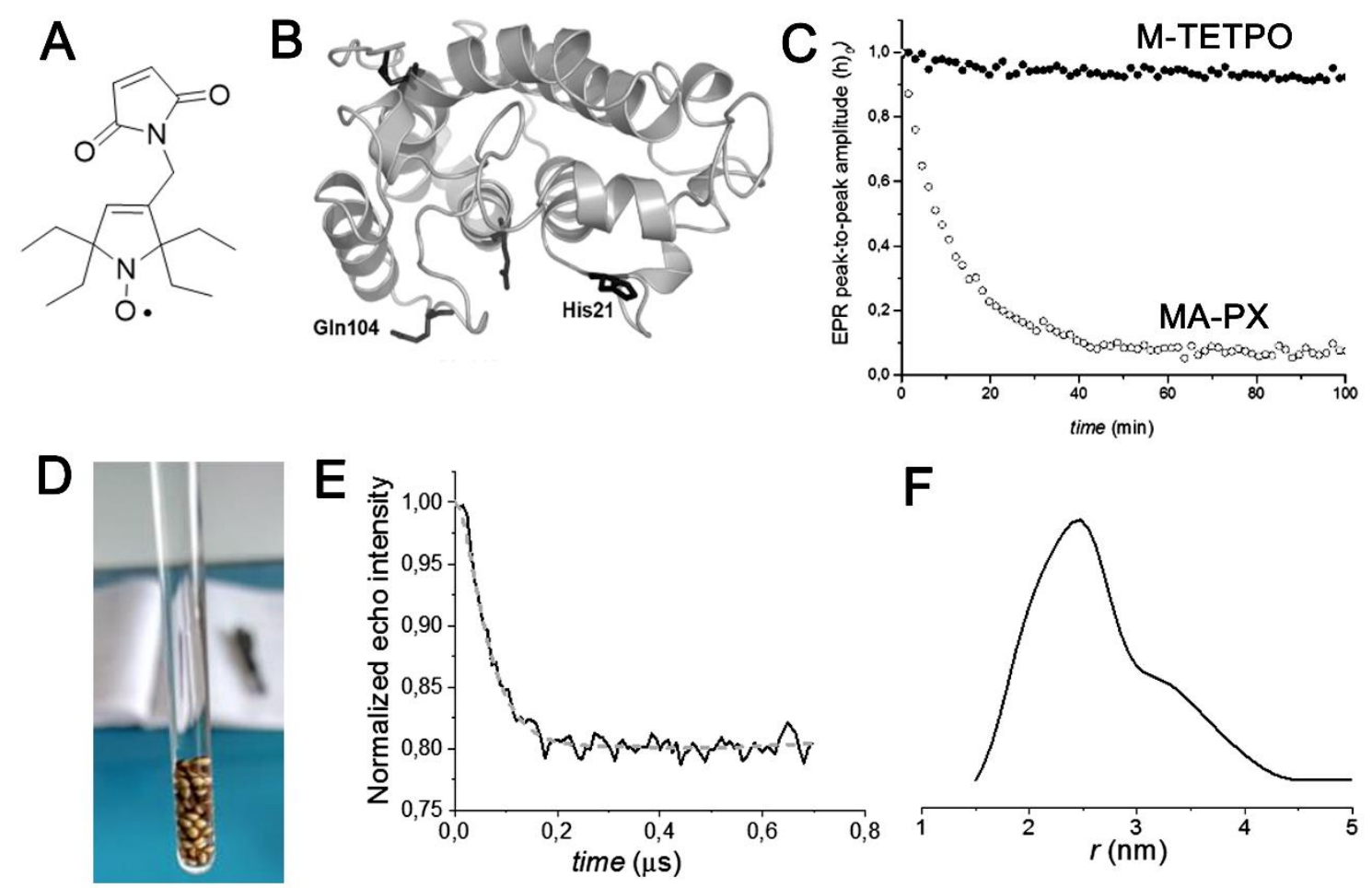

Fig.12: Application of a nitroxide-based spin label for in-cell EPR spectroscopy: A) Chemical structure of the bio-resistant gem-diethyl nitroxide (M-TETPO). B) Structural model of NarJ with in evidence the two positions selected as labelling sites for the nitroxides M-TETPO (His21Cys and Gln104Cys). C) Reduction profile for NarJ respectively labelled with MA-PX and the gem-diethyl nitroxide (M-TETPO) respectively in the presence of ascorbate $(4 \mathrm{mM})$. D) Images of the X.laevis oocytes previously microinjected with NarJ labelled with the gem-diethyl nitroxide (M-TETPO), inside the EPR tube E) Background corrected X-band DEER traces recorded for NarJ labelled with the gem-diethyl nitroxide (M-TETPO) into X.laevis oocytes F) Distance distributions obtained after 
Tikhonov regularization for NarJ labelled with the gem-diethyl nitroxide (M-TETPO) inside X.laevis oocytes (images was adapted from ref. [89], Copyright 2019 Wiley). 